\title{
Coupled MPS-modal superposition method for 2D nonlinear fluid-structure interaction problems with free surface
}

\author{
Zhe Sun $^{\mathrm{a}, *}$, K. Djidjeli ${ }^{\mathrm{a}}$, Jing T. Xing ${ }^{\mathrm{a}}$, Fai Cheng ${ }^{\mathrm{b}}$ \\ ${ }^{a}$ CED/FSI Group, FEE, University of Southampton B176, Boldrewood Campus, Burgess Road, Southampton SO16 7QF, UK \\ ${ }^{\mathrm{b}}$ Strategic Research E Technology Policy Group, Lloyd's Register Southampton Boldrewood Innovation Campus, Burgess Road, South- \\ ampton, S016 7QF, UK
}

\section{A R T I C L E I N F O}

\section{Article history:}

Received 6 March 2015

Received in revised form

7 October 2015

Accepted 11 December 2015

Available online 12 January 2016

Keywords:

Moving particle semi-implicit (MPS)

method

Fluid structure interaction (FSI)

Modal superposition

Free surface flow

Floating beam

Flexible wedge dropping

\begin{abstract}
A B S T R A C T
In this paper, a coupled MPS-modal superposition method is developed for 2D nonlinear fluid-structure interaction problems. In this method, the rigid-body and relatively small elastic deformation are coupled together, which considers the mutual effect between them. The elastic deformation of the structure is represented by a mode superposition formulation, which is more efficient compared with FEM, regardless of the size of the structure. For 2D cases, if the first three modes are chosen to represent the flexible deformation of the structure, it only results in a $6 \times 6$ matrix equation to be solved. For the fluid motion, the modified Moving Particle Semi-implicit (MPS) method, which significantly reduces the fluctuation of pressure calculation of the original MPS method, is used.

Two nonlinear problems, i.e. breaking-water-dam impacting a floating beam and flexible wedge slamming into the water are simulated to demonstrate the performance of the developed method. The numerical simulations show that this coupling model is capable of providing stable results that are generally in good agreement with the available experimental data. For the highly nonlinear case with very large rigid motions, the mutual effect between elastic deformation and rigid motions could accumulate to a relatively remarkable level shown by the curves of trajectories or acceleration history of the body mass centre. This also indicates the importance of mutual effect to analyse highly nonlinear FSI problems with large rigid-body motions and relatively small flexible deformation.
\end{abstract}

(c) 2015 Elsevier Ltd. All rights reserved.

\section{Introduction}

Meshless method, in contrast to its mesh-based counterpart, means the computational field is discretized with a set of independent particles without the topological constraints (meshes). This feature makes it very suitable for the simulation of problems with highly deformable boundaries such as free surface flow in marine engineering. A Lagrangian type meshless method, which is also referred to as a particle method, means the motion of each particle is totally determined by its physical velocity; makes this kind of meshless method much easier and straightforward to track the free surface location. Smoothed Particle Hydrodynamics (SPH) (Monaghan, 1994) and Moving Particle Semi-implicit (MPS) (Koshizuka and Oka, 1996) methods are both representative examples of the Lagrangian meshless method.

\footnotetext{
* Corresponding author.

E-mail addresses: zs2g12@soton.ac.uk, heusun09@gmail.com (Z. Sun), kdd@soton.ac.uk (K. Djidjeli), jtxing@soton.ac.uk (J.T. Xing), Fai.Cheng@lr.org (F. Cheng).
} 
The MPS method, since its development by Koshizuka (Koshizuka and Oka, 1996), has been successfully applied to various incompressible free surface flow problems (Khayyer and Gotoh, 2009, 2013). However, problems such as nonphysical pressure fluctuation also hinder further its application to Fluid Structure Interaction (FSI) problems. Many researchers have developed different techniques (Khayyer and Gotoh, 2009, 2013; Tsuruta et al., 2013; Lee et al., 2011) to improve its performance. The authors of this study have also proposed some modifications in (Sun et al., 2014, 2015). With all these improvements, the MPS method has been successfully applied to rigid-body FSI problems, for example, the wedge dropping (Sun et al., 2014, 2015; Akimoto, 2013), sloshing (Sun et al., 2014, 2015; Khayyer and Gotoh, 2011), and ship-wave interaction (Shibata et al., 2009, 2012) problems.

For FSI problems with flexible structures, Finite Element (FE) method has been used to calculate the structure dynamics, in combination with MPS method for the calculation of fluid motions (Sun et al., 2015; Lee et al., 2007).

For a typical ship structure, the overall motion could be described as a large rigid-body motion, such as, a combination of large forward motion, pitch, heave, and plus a relatively small flexible bending deformation. This feature makes that the modal superposition theorem adequate and quite suitable for representing this flexible deformation part. Moreover, it is also more efficient than its FE counterpart in terms of computational burden. In practice, considering the geometrically slimness of a ship structure, the beam model is normally adopted to describe its dynamics response to external excitations. Even for a non-beam like structure, for example a floating oil drilling platform, as long as the flexible motion part is relatively small, the modal superposition theorem is also applicable. What is worth mentioning here is that the mode function for a general shape structure is normally obtained from FE method rather than from analytical solution of beam theory, however this only requires one time prior calculation instead of solving FE model at each time step during the FSI computation.

Actually, this modal superposition technique has been successfully used to calculate interaction problems between water and large floating flexible structures (Jin and Xing, 2007; Kashiwagi, 2000; Newman, 1994). In these simulations, the fluid domain is computed by potential flow with linearized boundary conditions, and as such it could not handle the highly deformable free surface situations. For the structure part, either no rigid-body modes (Jin and Xing, 2007) or only some of them such as heave and pitch (Kashiwagi, 2000; Newman, 1994) are included in the computation, since the rigid-body motion part is very small and the elastic deformation is the main concern for these particular problems. In (Sun, 2007), the rigid-body motion and modal superposition are also coupled in the problem of elastic cylindrical shell entering water. This coupling is only in terms of force computation, which means that the force for rigid-body motion takes into account the effect of flexible deformation. It is not a "genuinely" coupling because the rigid-body force (i.e. total force and torque applied on the structure) will also affect the flexible kinetic parameters (i.e. generalized coordinates of each mode), as shown in Section 2.

Similarly, in ship Hydroelasticity (Bishop et al., 1986; Chen et al., 2006; Temarel and Hirdaris, 2009), this idea has also been extensively applied to compute the structure dynamics in regular and irregular waves (irregular wave calculation is also based on the regular case using spectral method). However, this computation is usually based on the small wave amplitude assumption (linear wave), and consequently the fluid domain is calculated using potential flow theory, subject to the boundary conditions at the mean free surface and structure positions (Temarel and Hirdaris, 2009). This means that both the rigid-body and flexible motion parts can only be relatively small in this method. Moreover, in the governing equations (Temarel and Hirdaris, 2009), there are no terms representing the mutual effect between rigid-body motion and flexible deformation, although the rigid and flexible variables are solved simultaneously. This way of handling FSI computations may be accurate enough for small motions, but in the case of violent water structure interaction, in which the rigid-body motion part is very large, the effect from structure flexibility to rigid-body motion should be considered and vice versa (which can be seen from the new structure governing equations in Section 2).

As a consequence, in this study a model which fully couples the rigid-body motion and small flexible deformation (by mode function) is developed to calculate the violent water and flexible structure interaction problems.

This model can be integrated with various fluid solvers, e.g. mesh-based or meshless ones. In this work, the modified MPS method (Sun et al., 2015) is employed to compute the fluid motion and the pressure imposed on structure. The interaction between fluid and structure solvers is conducted in a strong coupling manner, i.e. iterative until the convergence condition is satisfied.

The rest of this paper is organized as follows. First, the detailed derivation of the structural model for a general rigid body with flexible beams attached on it is given in Section 2. Based on this, the governing equations for three special cases, i.e. pure rigid body, floating flexible beam and symmetric wedge with flexible bottom are also derived. Then, the modified MPS method is briefly illustrated in Section 3. In Section 4, the water structure interaction strategy is presented. Finally, the results of the validation test cases, which include the break-dam hitting a free floating beam and the dropping of a wedge with flexible bottom, are discussed in Section 5.

\section{Structure solver-Dynamics of coupled rigid-body and flexible beams}

The coupled rigid-body and modal superposition (CRMS) model are developed in details in this section. In 2D model, the slim beam-like structure is quite common in ship industry, such as, the wall of the LNG container, the deck or even the whole ship structure. Consequently, the dynamics of the general equations for a rigid body with flexible beams attached to it is first derived. Based on this general assumption, the governing equations for three special cases are derived, i.e. pure rigid body, floating flexible beam (a reasonable approximation of ship structure) and wedge with flexible bottoms. 


\subsection{Kinetic description}

In Fig. 2.1, one rigid body with two flexible beam-like structures attached to it are investigated here. However, the proposed formulation is generally applicable for the cases with more beams. The beam-like structures are either firmly connected with the rigid-body if only one end is attached, or at least simply supported at both ends if they are all connected to the rigid-body. This kind of configuration could enforce that the beam-like structure will not freely rotate with respect to the rigid-body, which is a reasonable assumption for most of the physical situations in marine engineering. This means the relative position between these local systems (as will be defined below) will not change during the movement.

a) Coordinate systems

Two types of coordinate systems are used in this study, which include a global $X-Y$ system and body-attached (local) $x_{R}-y_{R}$ and $s_{i}-w_{i}$ systems ( $i=A, B$, represents the index of Beams $\mathrm{A}$ and $\mathrm{B}$, respectively).

The global $X-Y$ system is fixed in the space, whereas the body-attached (local) coordinate systems follow the translational and rotational motions of the corresponding components, but do not deform with the body if it is attached to the beam-like structure. The origins of these local coordinate systems are always chosen to be the mass centre of corresponding undeformed substructures.

b) Definition of the motion variables

The motion of the rigid-body part could be described by position of its mass centre $\boldsymbol{X}_{c R}=\left[X_{c R}(t), Y_{c R}(t)\right]^{T}$, which is the coordinate of the $x_{R}-y_{R}$ system origin (i.e., $O_{R}$ ) in global system, and the angle $\theta_{R}(t)$ from $O-X$ axis (anti-clockwise) to $O_{R}-X_{R}$ axis. For the flexible beam-like part, except for the mass centre $\left(O_{f A}\right.$ and $\left.O_{f B}\right)$ positions $\boldsymbol{X}_{c f i}=\left[X_{c f i}(t), Y_{c f i}(t)\right]^{T}$ and rotational angles $\theta_{f i}(t)$; additional variables $\eta_{i}\left(s_{i}, t\right)$ which describe the deflection of beam $i$ with respect to the corresponding beam central lines are required.

For describing the relation between rigid and flexible parts, the variables $\boldsymbol{x}_{o f i}=\left[x_{o f i}, y_{o f i}\right]^{T}$ are defined as the vectors from $O_{R}$ to $O_{f i}$, respectively. Compared with the aforementioned position vectors e.g. $\boldsymbol{X}_{c f}$, this $\boldsymbol{x}_{\text {ofi }}$ is represented in the $x_{R}-y_{R}$ system instead of the global $X-Y$ system. Because the relative positions between different local systems are unchanged during the motion, these vectors would be constants. Moreover, the angular differences between different systems $\beta_{i}$ are constants as well, i.e.

$$
\begin{aligned}
& \beta_{i}=\theta_{R}(t)-\theta_{f i}(t) \equiv \text { const } . \\
& \boldsymbol{x}_{\text {ofi }} \equiv \text { const. }
\end{aligned}
$$

Thus, their time derivatives of the angular variables are equal to each other, i.e.

$$
\begin{aligned}
& \dot{\theta}_{R}=\dot{\theta}_{f i} \\
& \ddot{\theta}_{R}=\ddot{\theta}_{f i}
\end{aligned}
$$

Similarly, $\boldsymbol{x}_{R}=\left[x_{R}, y_{R}\right]^{T}$ is defined as the vector from the mass centre of the rigid body $O_{R}$ to each point on the rigid body. And it is also represented in the local $x_{R}-y_{R}$ system. Hence, for each point, the corresponding representation of the vector would remain unchanged.

c) Kinetics of each point on structure

With the above definitions, the global coordinates of each point on rigid-body part, i.e. $\boldsymbol{X}_{R}=\left[X_{R}(t), Y_{R}(t)\right]^{T}$ could be represented as:

$$
\boldsymbol{X}_{R}=\boldsymbol{X}_{c R}+\boldsymbol{R}_{R} \boldsymbol{x}_{R}
$$

where $\boldsymbol{R}_{R}$ is the rotation matrix which relates the local $x_{R}-y_{R}$ system with the global $X-Y$ system. Its definition is given in Eq. (2.11). For the flexible beam-like structure, its motion could be described by the coordinates of the points on the central line $\boldsymbol{X}_{f i}=\left[X_{f i}(t), Y_{f i}(t)\right]^{T}$ as:

$$
\boldsymbol{X}_{f i}=\boldsymbol{X}_{c f i}+\boldsymbol{R}_{f i} \xi_{i}
$$

where $\xi_{i}$ is the coordinate of the points on $i^{\text {th }}$ beam's central line represented in the local $s_{i}-w_{i}$ system as:

$$
\xi_{i}=\left[s_{i}, \eta_{i}\right]^{T}
$$

According to the beam assumption, the deformation only occurs in the direction perpendicular to the beam central line, which means $s_{i}$ would not change for each point during the movement, i.e.

$$
\begin{aligned}
& \dot{\boldsymbol{\xi}}_{i}=\left[0, \dot{\eta}_{i}\right]^{T} \\
& \ddot{\boldsymbol{\xi}}_{i}=\left[0, \ddot{\eta}_{i}\right]^{T}
\end{aligned}
$$




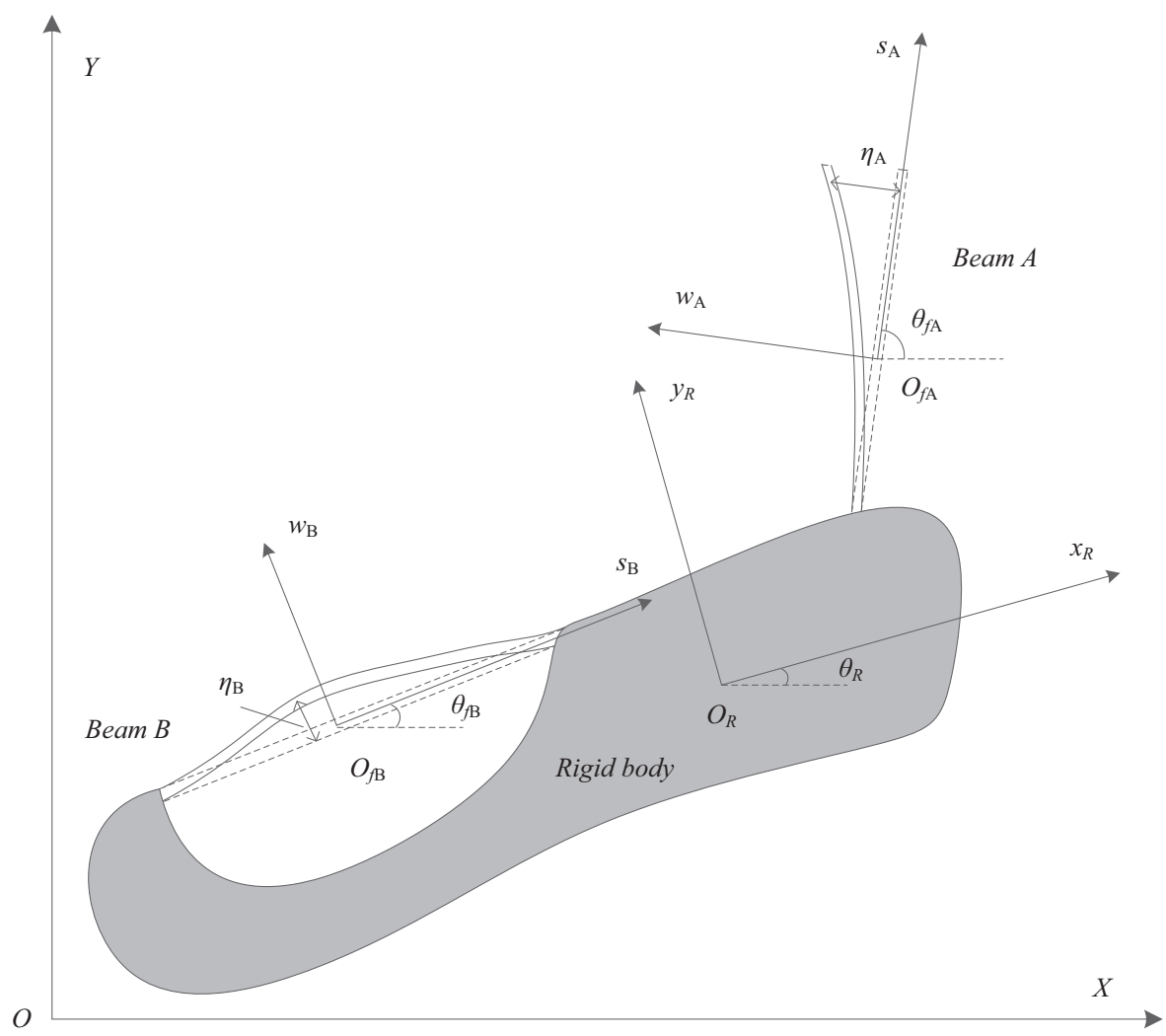

Fig. 2.1. Sketch of general rigid body with flexible beams system.

$\boldsymbol{X}_{c f i}$ could be related to $\boldsymbol{X}_{c R}$ as:

$$
\boldsymbol{X}_{c f i}=\boldsymbol{X}_{c R}+\boldsymbol{R}_{R} \boldsymbol{x}_{o f i}
$$

By submitting Eq. (2.9) into Eq. (2.5), $\boldsymbol{X}_{f i}$ could be expressed as:

$$
\boldsymbol{X}_{f i}=\boldsymbol{X}_{c R}+\boldsymbol{R}_{R} \boldsymbol{x}_{o f i}+\boldsymbol{R}_{f i} \xi_{i}
$$

Here, $\boldsymbol{R}_{f i}$ is similar to $\boldsymbol{R}_{R}$, and is also the rotation matrix which converts the coordinate in local $s_{i}-w_{i}$ system into the global $X-Y$ system. The definitions of $\boldsymbol{R}_{R}$ and $\boldsymbol{R}_{f i}$ are of the form:

$$
\boldsymbol{R}_{j}=\left[\begin{array}{cc}
\cos \theta_{j} & -\sin \theta_{j} \\
\sin \theta_{j} & \cos \theta_{j}
\end{array}\right]
$$

where $j$ indicates different local systems, i.e. rigid body, $R$; beam $A, f A$ or beam $\mathrm{B}, f B$. Their first and second order time derivatives, which will be used in the following derivation, are given as

$$
\begin{aligned}
& \dot{\boldsymbol{R}}_{j}=\boldsymbol{R}_{j} \boldsymbol{U} \dot{\theta}_{j} \\
& \ddot{\boldsymbol{R}}_{j}=\dot{\boldsymbol{R}}_{j} \boldsymbol{U} \dot{\theta}_{j}+\boldsymbol{R}_{j} \boldsymbol{U} \ddot{\theta}_{j}=-\boldsymbol{R}_{j} \dot{\theta}_{j}^{2}+\boldsymbol{R}_{j} \boldsymbol{U} \ddot{\theta}_{j}
\end{aligned}
$$

where $\boldsymbol{U}$ is introduced to simplify the derivation, and it is given by

$$
\boldsymbol{U}=\left[\begin{array}{cc}
0 & -1 \\
1 & 0
\end{array}\right]
$$

The following relationships of matrices $\boldsymbol{R}_{\mathbf{j}}$ and $\boldsymbol{U}$ would be used in the following derivations:

$$
\begin{aligned}
& \boldsymbol{R}_{j} \boldsymbol{R}_{j}^{T}=\boldsymbol{R}_{j}^{T} \boldsymbol{R}_{j}=\boldsymbol{U} \boldsymbol{U}^{T}=\boldsymbol{U}^{T} \boldsymbol{U}=\boldsymbol{I}_{2} \\
& \boldsymbol{U U}=\boldsymbol{U}^{T} \boldsymbol{U}^{T}=-\boldsymbol{I}_{2}
\end{aligned}
$$

where $\boldsymbol{I}_{2}$ is a $2 \times 2$ identity matrix. With these formulations, the velocity and acceleration of each point on the rigid body or beam-like structure could be derived as: 


$$
\begin{aligned}
\dot{\boldsymbol{X}}_{R} & =\dot{\boldsymbol{X}}_{c R}+\dot{\boldsymbol{R}}_{R} \boldsymbol{x}_{R}=\dot{\boldsymbol{X}}_{c R}+\boldsymbol{R}_{R} \boldsymbol{U} \dot{\theta}_{R} \boldsymbol{x}_{R} \\
\dot{\boldsymbol{X}}_{f i} & =\dot{\boldsymbol{X}}_{c R}+\dot{\boldsymbol{R}}_{R} \boldsymbol{x}_{o f i}+\dot{\boldsymbol{R}}_{f i} \xi_{i}+\boldsymbol{R}_{f i} \dot{\xi}_{i}=\dot{\boldsymbol{X}}_{c R}+\boldsymbol{R}_{R} \boldsymbol{U} \dot{\theta}_{R} \boldsymbol{x}_{o f i}+\boldsymbol{R}_{f i} \boldsymbol{U} \dot{\theta}_{f i} \xi_{i}+\boldsymbol{R}_{f i} \dot{\xi}_{i} \\
\ddot{\boldsymbol{X}}_{R} & =\ddot{\boldsymbol{X}}_{c R}+\ddot{\boldsymbol{R}}_{R} \boldsymbol{x}_{R}=\ddot{\boldsymbol{X}}_{c R}+\left(\boldsymbol{R}_{R} \boldsymbol{U} \ddot{\theta}_{R}-\boldsymbol{R}_{R} \dot{\theta}_{R}^{2}\right) \boldsymbol{x}_{R} \\
\ddot{\boldsymbol{X}}_{f i} & =\ddot{\boldsymbol{X}}_{c R}+\ddot{\boldsymbol{R}}_{R} \boldsymbol{x}_{o f i}+\ddot{\boldsymbol{R}}_{f i} \xi_{i}+2 \dot{\boldsymbol{R}}_{f i} \dot{\xi}_{i}+\boldsymbol{R}_{f i} \ddot{\xi}_{i}=\ddot{\boldsymbol{X}}_{c R}+\left(\boldsymbol{R}_{R} \boldsymbol{U} \ddot{\theta}_{R}-\boldsymbol{R}_{R} \dot{\theta}_{R}^{2}\right) \boldsymbol{x}_{o f i} \\
& +\left(\boldsymbol{R}_{f i} \boldsymbol{U} \ddot{\theta}_{f i}-\boldsymbol{R}_{f i} \dot{\theta}_{f i}^{2}\right) \xi_{i}+2 \boldsymbol{R}_{f i} \boldsymbol{U} \dot{\theta}_{f i} \dot{\xi}_{i}+\boldsymbol{R}_{f i} \ddot{\xi}_{i}
\end{aligned}
$$

In the computation, the perimeter of the whole structure is represented by a set of discrete points. Each point on the rigid-body part could be defined by Eq. (2.4), after the related variables are obtained. For the beam-like structure part, Eq. (2.10) only gives the position of the points on its central line. The points on its perimeter are determined in the following way:

The coordinates of each point is updated by linearly combining the rigid and flexible parts. For the rigid part, the position is simply determined as a normal rigid-body. The flexible deformation is updated based on the assumption that the beam is made up by multiple layers of materials and each layer will undergo the same deformation around its own central line. More specifically, for the points that are not on the beam central line, the flexible deformation is obtained by shifting the corresponding value from the layer that is on the beam central line.

\subsection{Modal superposition approach}

By using the modal superposition method, the small elastic deflection part $\eta_{i}\left(s_{i}, t\right)$ could be represented as:

$$
\eta_{i}\left(s_{i}, t\right)=\boldsymbol{\Phi}_{i}^{T} \boldsymbol{q}_{i}
$$

where $\boldsymbol{\Phi}_{i}$ and $\boldsymbol{q}_{i}$ are the vectors of mode functions and the corresponding generalised coordinates. They are defined as follows:

$$
\begin{aligned}
\boldsymbol{\Phi}_{i} & =\left[\phi_{i 1}(s), \phi_{i 2}(s), \ldots, \phi_{i n}(s)\right]^{T} \\
\boldsymbol{q}_{i} & =\left[q_{i 1}(t), q_{i 2}(t), \ldots, q_{i n}(t)\right]^{T}
\end{aligned}
$$

Separating the spatial and time variables in Eq. (2.21), the time derivatives of $\eta_{i}\left(s_{i}, t\right)$ could be further expressed as:

$$
\begin{aligned}
\dot{\eta}_{i} & =\boldsymbol{\Phi}_{i}^{T} \dot{\boldsymbol{q}}_{i} \\
\ddot{\eta}_{i} & =\boldsymbol{\Phi}_{i}^{T} \ddot{\boldsymbol{q}}_{i}
\end{aligned}
$$

The mode functions are obtained based on the Euler beam equation with particular boundary conditions, as required in different problems. Furthermore, the orthogonal relationships should also be satisfied, that is,

$$
\begin{aligned}
& \int_{f i} \boldsymbol{\Phi}_{i} \boldsymbol{\Phi}_{i}^{T} \rho_{l i} d s=\boldsymbol{I}_{n} \\
& \int\left(\frac{d^{2} \boldsymbol{\Phi}_{i}}{d s_{i}^{2}}\right) E_{i} J_{i}\left(\frac{d^{2} \boldsymbol{\Phi}_{i}}{d s_{i}^{2}}\right)^{T} d s=\boldsymbol{\Lambda}_{i}, \boldsymbol{\Lambda}_{i}=\operatorname{diag}\left(\omega_{i k}{ }^{2}\right)
\end{aligned}
$$

where $\omega_{i k}$ represents the $k^{\text {th }}$ natural circular frequency of the $i^{\text {th }}$ beam, and $E_{i}, \rho_{l i}$ and $J_{i}$ are Young's module, the line density and the 2 nd moment of the $i^{\text {th }}$ beam's cross section, respectively. $\boldsymbol{I}_{n}$ is the $n \times n$ identity matrix, where $n$ is the number of modes that is used. The line integration is conducted along the neutral line of the beam.

Introducing the modal superposition model makes it possible to use the generalized coordinates $\boldsymbol{q}_{i}$ to represent the flexible deformation of the beam. This would enable us to use the desirable orthogonal properties of Eqs. (2.26) and (2.27) to further simplify the form of these equations in the following content.

In this work, two different set of mode functions are employed for the two problems, i.e. free floating beam on free surface and wedge with flexible bottoms.

For the free floating beam case, the corresponding boundary condition at both ends is free. The mode functions used in (Jin and Xing, 2007) is employed here. The first two rigid modes (heave and pitch modes) are eliminated since these parts will be taken into account by the rigid-body motion. In the following equation for mode function, the index number $i$ is omitted since they are used for a general formulation instead of a special case for a particular beam:

$$
\phi_{k}(s)=\left\{\begin{array}{l}
\frac{1}{\sqrt{M_{f}}}\left[\frac{\cosh \left(\mu_{k} s / a\right)}{\cosh \left(\mu_{k}\right)}+\frac{\cos \left(\mu_{k} s / a\right)}{\cos \left(\mu_{k}\right)}\right], k=1,3,5, \ldots \\
\frac{1}{\sqrt{M_{f}}}\left[\frac{\sinh \left(\mu_{k} s / a\right)}{\sinh \left(\mu_{k}\right)}+\frac{\sin \left(\mu_{k} s / a\right)}{\sin \left(\mu_{k}\right)}\right], k=2,4,6, \ldots
\end{array}\right.
$$


where $M_{f}$ is the total mass of the beam, $a=L / 2$ is half-length of the beam, and $\mu_{k}$ are the positive real roots of the eigenvalue equations:

$$
\left\{\begin{array}{l}
\tan \left(\mu_{k}\right)+\tanh \left(\mu_{k}\right)=0, k=1,3,5, \ldots \\
\tan \left(\mu_{k}\right)-\tanh \left(\mu_{k}\right)=0, k=2,4,6, \ldots
\end{array}\right.
$$

The first three roots of these equations are: $\mu_{1}=2.3650, \mu_{2}=3.9266, \mu_{3}=5.4978$.

In the case of wedge with flexible bottoms, each of the symmetrically installed bottoms is modelled as a cantilever beam, which means the boundary condition at the ends is fixed-free. The corresponding mode function is:

$$
\phi_{k}(S)=\frac{1}{\sqrt{M_{f}}}\left[\cosh \left(\frac{\mu_{k} S}{a}\right)-\cos \left(\frac{\mu_{k} S}{a}\right)-\sigma_{k}\left(\sinh \left(\frac{\mu_{k} S}{a}\right)-\sin \left(\frac{\mu_{k} S}{a}\right)\right)\right], k=1,2,3,
$$

where $\sigma_{k}$ is defined as $\sigma_{k}=\frac{\sin \left(2 \mu_{k}\right)-\sinh \left(2 \mu_{k}\right)}{\cos \left(2 \mu_{k}\right)-\cosh \left(2 \mu_{k}\right)} ; \mu_{k}$ are the positive real roots of the following equations:

$$
\cos \left(2 \mu_{k}\right)+\cosh \left(2 \mu_{k}\right)+1=0, k=1,2,3, \ldots
$$

The first three roots are: $\mu_{1}=0.9375, \mu_{2}=2.3470, \mu_{3}=3.9274$.

To simplify the form of the equations derived in Section 2.3 , the following definitions are introduced

$$
\begin{gathered}
\boldsymbol{\psi}_{0}=\left[\psi_{01}, \psi_{02}, \psi_{03}, \ldots\right]^{T}=\int \boldsymbol{\Phi} \rho_{l} d s, \\
\boldsymbol{\psi}_{1}=\left[\psi_{11}, \psi_{12}, \psi_{13}, \ldots\right]^{T}=\int s \boldsymbol{\Phi} \rho_{l} d s,
\end{gathered}
$$

\subsection{General governing equations}

Using Sections 2.1 and 2.2, the motion of the structure could be fully described by the generalised position variables $\boldsymbol{D}$ and the corresponding generalised force $\boldsymbol{Q}$ variables as follows:

$$
\begin{aligned}
& \boldsymbol{D}=\left[\boldsymbol{X}_{c R}^{T}, \theta, \boldsymbol{q}_{A}^{T}, \boldsymbol{q}_{B}^{T}, \ldots\right]^{T} \\
& \boldsymbol{Q}=\left[\mathbf{Q}_{X_{c R}}^{T}, Q_{\theta}, \mathbf{Q}_{q_{A}}^{T}, \boldsymbol{Q}_{q_{B}}^{T}, \ldots\right]^{T}
\end{aligned}
$$

As shown in Eq. (2.1), $\theta_{R}$ and $\theta_{f i}$ are not independent to each other, therefore for simplicity, the angle variable $\theta$ in $\boldsymbol{D}$ is selected to be the rigid rotational angle $\theta_{R}$ without affecting the structure of the formulations. Moreover, although the time derivatives of $\theta_{R}$ and $\theta_{f i}$ are the same (i.e. Eqs. (2.2) and (2.3)), they are still annotated differently in the following derivations in order to show clearly the origin of each term. The variables $\boldsymbol{q}_{i}$ are the generalised coordinates for Beam A or Beam B, respectively, which are defined by Eq. (2.23).

The force variables $\boldsymbol{Q}_{X_{c R}}, Q_{\theta}$ and $\boldsymbol{Q}_{q_{i}}$ are non-conservative forces corresponding to the rigid-body motion part (i.e. $\boldsymbol{X}_{c R}$ and $\theta$ ) and the elastic parts (i.e. $\boldsymbol{q}_{i}$ ), respectively. The vector type forces are defined as:

$$
\begin{aligned}
& \boldsymbol{Q}_{X_{c R}}=\left[Q_{X_{c R}}, Q_{Y_{c R}}\right]^{T} \\
& \boldsymbol{Q}_{q_{i}}=\left[Q_{q i 1}, Q_{q i 2}, \ldots, Q_{q i n}\right]^{T}
\end{aligned}
$$

According to the principle of Lagrange Mechanics, the motion of the structure could be described as:

$$
\begin{aligned}
& \boldsymbol{Q}_{X_{c R}}=\frac{d}{d t}\left(\frac{\partial T}{\partial \dot{\boldsymbol{X}}_{c R}}\right)+\frac{\partial V}{\partial \boldsymbol{X}_{c R}}-\frac{\partial T}{\partial \boldsymbol{X}_{c R}} \\
& Q_{\theta}=\frac{d}{d t}\left(\frac{\partial T}{\partial \dot{\theta}}\right)+\frac{\partial V}{\partial \theta}-\frac{\partial T}{\partial \theta} \\
& \boldsymbol{Q}_{q_{i}}=\frac{d}{d t}\left(\frac{\partial T}{\partial \dot{\boldsymbol{q}}_{i}}\right)+\frac{\partial V}{\partial \boldsymbol{q}_{i}}-\frac{\partial T}{\partial \boldsymbol{q}_{i}}
\end{aligned}
$$

where $T$ and $V$ are kinetic and potential energies of the whole structure, respectively. The kinetic energies for the rigid body $\left(T_{R}\right)$ and flexible $\left(T_{f i}\right)$ beams; and the whole structure are given by:

$$
\begin{aligned}
& T_{f i}=\frac{1}{2} \int_{f i} \dot{\boldsymbol{X}}_{f i}^{T} \rho_{l} \dot{\boldsymbol{X}}_{f i} d s \\
& T_{R}=\frac{1}{2} \iint_{R} \dot{\boldsymbol{X}}_{R}^{T} \rho \dot{\boldsymbol{X}}_{R} d x d y
\end{aligned}
$$




$$
T=T_{R}+\sum_{i} T_{f i}
$$

For the potential energies, they are given by:

$$
\begin{aligned}
V_{R} & =M_{R} g Y_{c R} \\
V_{f i} & =\frac{1}{2} \int_{f i} \frac{d^{2} \eta_{f i}}{d s^{2}} E_{i} J_{i} \frac{d^{2} \eta_{f i}}{d s^{2}} d s+M_{f i} g Y_{c f i}=\frac{1}{2} \boldsymbol{q}_{i}^{T}\left(\int\left(\frac{d^{2} \boldsymbol{\Phi}_{i}}{d s^{2}}\right) E_{i} J_{i}\left(\frac{d^{2} \boldsymbol{\Phi}_{i}}{d s^{2}}\right)^{T} d s\right) \boldsymbol{q}_{i}+M_{f i} g Y_{c f i} \\
& =\frac{1}{2} \boldsymbol{q}_{i}^{T} \boldsymbol{\Lambda}_{i} \boldsymbol{q}_{i}+M_{f i} g\left(Y_{c R}+\sin \theta_{R} x_{o f i}+\cos \theta_{R} y_{o f i}\right) \\
V & =V_{R}+\sum_{i} V_{f i}
\end{aligned}
$$

where $V_{R}$ and $V_{f i}$ are the rigid body and flexible beams potential energies, respectively, and $M_{R}$ and $M_{f i}$ are the masses for the corresponding parts.

The generalised forces corresponding to the rigid-body motion (i.e. $\boldsymbol{X}_{c R}$ and $\theta$ ) and the elastic ones (i.e. $\boldsymbol{q}_{i}$ ) could be determined using the virtual work principle. This is achieved by establishing an equation that the virtual work done by the generalised forces should be equal to the one done by active external non-conservative forces through generalised virtual displacements. In this work, we assume that the whole structure is subject to distributed pressure $p$ only (e.g. water pressure). No concentrated force or moment is applied on it. Hence, the following equations are obtained:

$$
\begin{aligned}
& \delta \boldsymbol{X}_{c R}^{T} \boldsymbol{Q}_{X_{c R}}=\delta \boldsymbol{X}_{c R}^{T} \oint_{\text {all }}(p \boldsymbol{n}) d l \\
& \delta \theta Q_{\theta}=\delta \theta \oint_{\text {all }} p\left(X_{p} n_{y}-Y_{p} n_{x}\right) d l \\
& \delta \boldsymbol{q}_{i}^{T} \boldsymbol{Q}_{q_{i}}=\oint_{f i}\left[(p \boldsymbol{n}) \cdot \boldsymbol{e}_{i w}\right] \delta \eta_{f i} d l=\delta \boldsymbol{q}_{i}^{T} \oint_{f i}\left[(p \boldsymbol{n}) \cdot \boldsymbol{e}_{i w}\right] \boldsymbol{\Phi}_{i} d l
\end{aligned}
$$

where $\boldsymbol{n}=\left[n_{x}, n_{y}\right]^{T}$ is the unit normal vector of points on the perimeter of the whole surface, which points towards the interior structural domain (i.e., outside the fluid domain if fluid is in the vicinity). $\boldsymbol{X}_{p}=\left[X_{p}, Y_{p}\right]^{T}$ are the vectors pointing from $O_{R}$ to the points on the perimeter of the whole structure. $\boldsymbol{e}_{i w}=\left[e_{i w 1}, e_{i w 2}\right]^{\mathrm{T}}$ is the unit vector of the $w_{i}$ direction. All these vectors are represented in the global $X-Y$ system. The integrations with subscripts all or fi mean that the calculations are conducted on the perimeter of the whole structure or just on the corresponding beams.

Thus, the generalised forces are:

$$
\begin{aligned}
& \boldsymbol{Q}_{X_{c R}}=\oint_{\text {all }} p \boldsymbol{n} d l \\
& Q_{\theta}=\oint_{\text {all }} p\left(X_{p} n_{y}-Y_{p} n_{x}\right) d l \\
& \boldsymbol{Q}_{q_{i}}=\oint_{f i}\left[(p \boldsymbol{n}) \cdot \boldsymbol{e}_{i w}\right] \boldsymbol{\Phi}_{i} d l
\end{aligned}
$$

After substituting $T, V, \boldsymbol{Q}_{X_{c R}}, Q_{\theta}$ and $\boldsymbol{Q}_{q_{i}}$ into the Lagrange equation, and with some tedious derivations using the chain rule, the governing equations for the coupled rigid-body and flexible beams system become:

$$
\begin{aligned}
& \sum_{i} \int_{f i}\left(\ddot{\boldsymbol{X}}_{c R}-\boldsymbol{R}_{R} \dot{\theta}_{R}^{2} \boldsymbol{x}_{o f i}+\boldsymbol{R}_{R} \boldsymbol{U} \ddot{\theta}_{R} \boldsymbol{x}_{o f i}-\boldsymbol{R}_{f i} \dot{\theta}_{f i}^{2} \boldsymbol{\xi}_{i}+\boldsymbol{R}_{f i} \boldsymbol{U} \ddot{\theta}_{f i} \boldsymbol{\xi}_{i}+2 \boldsymbol{R}_{f i} \boldsymbol{U} \dot{\theta}_{f i} \dot{\boldsymbol{\xi}}_{i}+\boldsymbol{R}_{f i} \ddot{\xi}_{i}\right) \rho_{l} d s \\
& +\iint_{R}\left(\ddot{\boldsymbol{X}}_{c R}-\boldsymbol{R}_{R} \dot{\theta}_{R}^{2} \boldsymbol{x}_{R}+\boldsymbol{R}_{R} \boldsymbol{U} \ddot{\theta}_{R} \boldsymbol{x}_{R}\right) \rho d x d y+\left(M_{R}+\sum_{i} M_{f i}\right) g\left[\begin{array}{l}
0 \\
1
\end{array}\right]=\boldsymbol{Q}_{X_{c R}} \\
& \sum_{i} \int_{f i}\left(\ddot{\boldsymbol{X}}_{c R}^{T}+\ddot{\theta}_{R} \boldsymbol{x}_{o f i}{ }^{T} \boldsymbol{U}^{T} \boldsymbol{R}_{R}^{T}-\dot{\theta}_{R}^{2} \boldsymbol{x}_{o f i}{ }^{T} \boldsymbol{R}_{R}^{T}+\ddot{\theta}_{f i} \boldsymbol{\xi}_{i}{ }^{T} \boldsymbol{U}^{\boldsymbol{T}} \boldsymbol{R}_{f i}^{T}+2 \dot{\theta}_{f i} \dot{\xi}_{i}{ }^{T} \boldsymbol{U}^{T} \boldsymbol{R}_{f i}^{T}-\dot{\theta}_{f i}^{2} \boldsymbol{\xi}_{i}{ }^{T} \boldsymbol{R}_{f i}^{T}+\ddot{\boldsymbol{\xi}}_{i}{ }^{T} \boldsymbol{R}_{f i}^{T}\right)\left(\boldsymbol{R}_{R} \boldsymbol{U} \boldsymbol{x}_{o f i}+\boldsymbol{R}_{f i} \boldsymbol{U} \xi_{i}\right) \rho_{l} d s \\
& +\iint_{R}\left(\ddot{\boldsymbol{X}}_{c R}^{T}-\dot{\theta}_{R}^{2} \boldsymbol{x}_{R}^{T} \boldsymbol{R}_{R}^{T}+\ddot{\theta}_{R} \boldsymbol{x}_{R}^{T} \boldsymbol{U}^{T} \boldsymbol{R}_{R}^{T}\right)\left(\boldsymbol{R}_{R} \boldsymbol{U} \boldsymbol{x}_{R}\right) \rho d x d y+\sum_{i} M_{f i}\left(\cos \theta_{R} x_{o f i 1}-\sin \theta_{R} x_{o f i 2}\right)=Q_{\theta} \\
& \int_{f i}\left(\ddot{\boldsymbol{X}}_{c R}^{T}+\ddot{\theta}_{R} \boldsymbol{x}_{o f i}{ }^{T} \boldsymbol{U}^{T} \boldsymbol{R}_{R}^{T}-\dot{\theta}_{R}^{2} \boldsymbol{x}_{o f i}{ }^{T} \boldsymbol{R}_{R}^{T}+\ddot{\theta}_{f i} \boldsymbol{\xi}_{i}^{T} \boldsymbol{U}^{T} \boldsymbol{R}_{f i}^{T}+2 \dot{\theta}_{f i} \dot{\boldsymbol{\xi}}_{i}^{T} \boldsymbol{U}^{T} \boldsymbol{R}_{f i}^{T}-\dot{\theta}_{f i}^{2} \boldsymbol{\xi}_{i}^{T} \boldsymbol{R}_{f i}^{T}+\ddot{\xi}_{i}^{T} \boldsymbol{R}_{f i}^{T}\right)\left(\boldsymbol{R}_{f i}\left[\begin{array}{c}
0 \\
1
\end{array}\right] \boldsymbol{\Phi}_{i}\right) \rho_{l} d s+\boldsymbol{\Lambda}_{i} \boldsymbol{q}_{i}=\boldsymbol{Q}_{q_{i}}
\end{aligned}
$$

For the rigid body part, the following integrations hold:

$$
\iint_{R} \rho d x d y=M_{R}
$$

$\iint_{R} \rho \boldsymbol{R}_{R} \boldsymbol{x}_{R} d x d y=0$ 


$$
\iint_{R} \boldsymbol{x}_{R}^{T} \boldsymbol{x}_{R} \rho d x d y=I_{R}
$$

which also means:

$$
\iint_{R} \rho \boldsymbol{R}_{R} \boldsymbol{U} \boldsymbol{x}_{R} d x d y=0
$$

where $I_{R}$ is the rotational inertia of the rigid body. The integration for the rigid-body part could be simplified as:

$$
\begin{gathered}
\iint_{R}\left(\ddot{\boldsymbol{X}}_{c R}-\boldsymbol{R}_{R} \dot{\theta}_{R}^{2} \boldsymbol{x}_{R}+\boldsymbol{R}_{R} \boldsymbol{U} \ddot{\theta}_{R} \boldsymbol{x}_{R}\right) \rho d x d y=\ddot{\boldsymbol{X}}_{c R} \iint_{R} \rho d x d y-\dot{\theta}_{R}^{2} \iint_{R} \rho \boldsymbol{R}_{R} \boldsymbol{x}_{R} d x d y+\ddot{\theta}_{R} \iint_{R} \rho \boldsymbol{R}_{R} \boldsymbol{U} \boldsymbol{x}_{R} d x d y=M_{R} \ddot{\boldsymbol{X}}_{c R} \\
\iint_{R}\left(\ddot{\boldsymbol{X}}_{c R}^{T}-\dot{\theta}_{R}^{2} \boldsymbol{x}_{R}^{T} \boldsymbol{R}_{R}^{T}+\ddot{\theta}_{R} \boldsymbol{x}_{R}^{T} \boldsymbol{U}^{T} \boldsymbol{R}_{R}^{T}\right)\left(\boldsymbol{R}_{R} \boldsymbol{U} \boldsymbol{x}_{R}\right) \rho d x d y=\ddot{\boldsymbol{X}}_{c R}^{T} \iint_{R} \rho \boldsymbol{R}_{R} \boldsymbol{U} \boldsymbol{x}_{R} d x d y+\ddot{\theta}_{R} \iint_{R} \boldsymbol{x}_{R}^{T} \boldsymbol{U}^{T} \boldsymbol{R}_{R}^{T} \boldsymbol{R}_{R} \boldsymbol{U} \boldsymbol{x}_{R} \rho d x d y \\
-\dot{\theta}_{R}^{2} \iint_{R} \boldsymbol{x}_{R}^{T} \boldsymbol{R}_{R}^{T} \boldsymbol{R}_{R} \boldsymbol{U} \boldsymbol{x}_{R} \rho d x d y=I_{R} \ddot{\theta}_{R}
\end{gathered}
$$

Finally, by substituting Eqs. (2.60) and (2.61) into Eqs. (2.53)-(2.55), the governing equations for the whole structure become:

$$
\begin{aligned}
& \sum_{i} \int_{f i}\left(\ddot{\boldsymbol{X}}_{c R}-\boldsymbol{R}_{R} \dot{\theta}_{R}^{2} \boldsymbol{x}_{o f i}+\boldsymbol{R}_{R} \boldsymbol{U} \ddot{\theta}_{R} \boldsymbol{x}_{o f i}-\boldsymbol{R}_{f i} \dot{\theta}_{f i}^{2} \boldsymbol{\xi}_{i}+\boldsymbol{R}_{f i} \ddot{U}_{f i} \xi_{i}+2 \boldsymbol{R}_{f i} \boldsymbol{U} \dot{\theta}_{f i} \dot{\xi}_{i}+\boldsymbol{R}_{f i} \ddot{\xi}_{i}\right) \rho_{l} d s+M_{R} \ddot{\boldsymbol{X}}_{c R}+\left(M_{R}+\sum_{i} M_{f i}\right) g\left[\begin{array}{l}
0 \\
1
\end{array}\right]=\boldsymbol{Q}_{X_{c R}} \\
& \sum_{i} \int_{f i}\left(\ddot{\boldsymbol{X}}_{c R}^{T}+\ddot{\theta}_{R} \boldsymbol{x}_{o f i}^{T} \boldsymbol{U}^{T} \boldsymbol{R}_{R}^{T}-\dot{\theta}_{R}^{2} \boldsymbol{x}_{o f i}{ }^{T} \boldsymbol{R}_{R}^{T}+\ddot{\theta}_{f i} \boldsymbol{\xi}_{i}^{T} \boldsymbol{U}^{T} \boldsymbol{R}_{f i}^{T}+2 \dot{\theta}_{f i} \dot{\boldsymbol{\xi}}_{i}^{T} \boldsymbol{U}^{\boldsymbol{T}} \boldsymbol{R}_{f i}^{T}-\dot{\theta}_{f i}^{2} \boldsymbol{\xi}_{i}^{T} \boldsymbol{R}_{f i}^{T}+\ddot{\xi}_{i}^{T} \boldsymbol{R}_{f i}^{T}\right)\left(\boldsymbol{R}_{R} \boldsymbol{U} \boldsymbol{x}_{o f i}^{R}+\boldsymbol{R}_{f i} \boldsymbol{U} \boldsymbol{\xi}_{i}\right) \rho_{l} d s+I_{R} \ddot{\theta}_{R} \\
& \quad+\sum_{i} M_{f i}\left(\cos \theta_{R} x_{o f i}-\sin \theta_{R} y_{o f i}\right)=Q_{\theta} \\
& \int_{f i}\left(\ddot{\boldsymbol{X}}_{c R}^{T}+\ddot{\theta}_{R} \boldsymbol{x}_{o f i}^{T} \boldsymbol{U}^{T} \boldsymbol{R}_{R}^{T}-\dot{\theta}_{R}^{2} \boldsymbol{x}_{o f i}{ }^{T} \boldsymbol{R}_{R}^{T}+\ddot{\theta}_{f i} \boldsymbol{\xi}_{i}^{T} \boldsymbol{U}^{T} \boldsymbol{R}_{f i}^{T}+2 \dot{\theta}_{f i} \dot{\boldsymbol{\xi}}_{i}^{T} \boldsymbol{U}^{T} \boldsymbol{R}_{f i}^{T}-\dot{\theta}_{f i}^{2} \boldsymbol{\xi}_{i}^{T} \boldsymbol{R}_{f i}^{T}+\ddot{\xi}_{i}^{T} \boldsymbol{R}_{f i}^{T}\right)\left(\boldsymbol{R}_{f i}\left[\begin{array}{l}
0 \\
1
\end{array}\right] \boldsymbol{\Phi}_{i}\right) \rho_{l} d s+\boldsymbol{\Lambda}_{i} \boldsymbol{q}_{i}=\boldsymbol{Q}_{q_{i}}
\end{aligned}
$$

\subsubsection{Special cases}

2.3.1.1. Rigid-body. If the object under consideration is just a rigid body, all the integrations corresponding to flexible beams in the above general governing equations would vanish. This means the final equations would simply reduce to the following form:

$$
\begin{aligned}
& M_{R} \ddot{\boldsymbol{X}}_{c R}+M_{R} g\left[\begin{array}{l}
0 \\
1
\end{array}\right]=\boldsymbol{Q}_{X_{c R}} \\
& I_{R} \ddot{\theta}_{R}=Q_{\theta}
\end{aligned}
$$

These equations are obviously identical to the classical rigid-body dynamics results, as expected.

2.3.1.2. Floating flexible beam. The case of a homogeneous floating flexible beam is studied in this section. The beam assumption is a common practice in marine engineering when describing the overall Hydroelasticity property of a ship structure. However, as mentioned before, the traditional Hydroelasticity theory actually performs the calculation in the way that rigid-body motion and flexible deformation are calculated separately without considering the mutual interaction effect, i.e. first computing the overall motion with rigid-body assumption and then calculating the flexible deformation with modal superposition technique based on the fluid force from rigid-body computation. The model proposed here provides the possibility to compute the coupled effect of rigid-body and flexible motions.

As shown in Fig. 2.2, there is only one flexible beam in this case. The subscript $i(i=A, B)$, which indicates different beams, could be omitted in the above equations. Furthermore, since the purely rigid body part does not exist in this case, the rigidbody system $x_{R}-y_{R}$ and flexible beam system $x_{f}-y_{f}$ coincide with each other, which lead to the following relations:

$$
\left\{\begin{array}{c}
\boldsymbol{x}_{o f} \equiv 0(a) \\
\theta_{R} \equiv \theta_{f}(b) \\
\boldsymbol{R}_{R} \equiv \boldsymbol{R}_{f}(c) \\
\boldsymbol{X}_{c R} \equiv \boldsymbol{X}_{c f}(d)
\end{array}\right.
$$

Substituting the above relations into the general governing equations Eqs. (2.62)-(2.64), lead to the following simplified expression for this case:

$$
\int_{f}\left(\ddot{\boldsymbol{X}}_{c f}-\boldsymbol{R}_{f} \dot{\theta}_{f}^{2} \boldsymbol{\xi}+\boldsymbol{R}_{f} \boldsymbol{U} \ddot{\theta}_{f} \boldsymbol{\xi}+2 \boldsymbol{R}_{f} \boldsymbol{U} \dot{\theta}_{f} \dot{\boldsymbol{\xi}}+\boldsymbol{R}_{f} \ddot{\xi}\right) \rho_{l} d s+M_{f} g\left[\begin{array}{l}
0 \\
1
\end{array}\right]=\boldsymbol{Q}_{X_{c f}}
$$




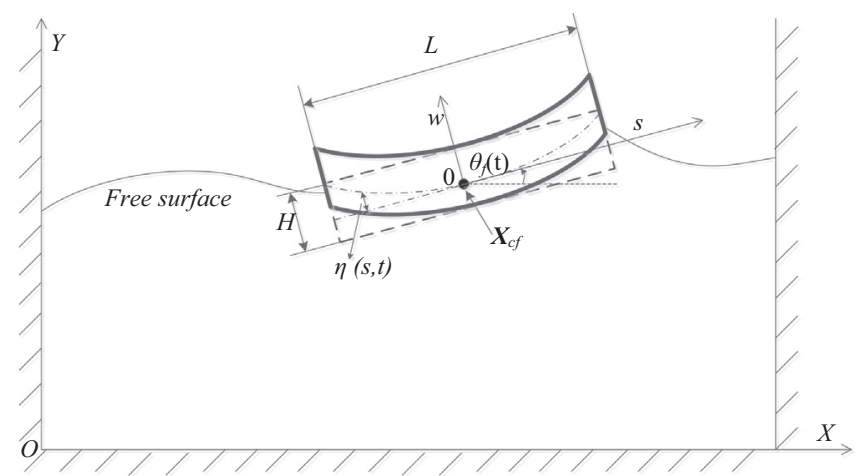

Fig. 2.2. Sketch of the flexible floating beam system.

$$
\begin{aligned}
& \int_{f}\left(\ddot{\boldsymbol{X}}_{c f}^{T}+\ddot{\theta}_{f} \xi^{T} \boldsymbol{U}^{\boldsymbol{T}} \boldsymbol{R}_{f}^{T}+2 \dot{\theta}_{f} \dot{\boldsymbol{\xi}}^{T} \boldsymbol{U}^{\boldsymbol{T}} \boldsymbol{R}_{f}^{T}-\dot{\theta}_{f}^{2} \boldsymbol{\xi}^{T} \boldsymbol{R}_{f}^{T}+\ddot{\boldsymbol{\xi}}^{T} \boldsymbol{R}_{f}^{T}\right)\left(\boldsymbol{R}_{f} U \boldsymbol{\xi}\right) \rho_{l} d s=Q_{\theta} \\
& \int_{f}\left(\ddot{\boldsymbol{X}}_{c f}^{T}+\ddot{\theta}_{f} \boldsymbol{\xi}^{T} \boldsymbol{U}^{\boldsymbol{T}} \boldsymbol{R}_{f}^{T}+2 \dot{\theta}_{f} \dot{\boldsymbol{\xi}}^{T} \boldsymbol{U}^{\boldsymbol{T}} \boldsymbol{R}_{f}^{T}-\dot{\theta}_{f}^{2} \boldsymbol{\xi}^{T} \boldsymbol{R}_{f}^{T}+\ddot{\boldsymbol{\xi}}^{T} \boldsymbol{R}_{f}^{T}\right)\left(\boldsymbol{R}_{f}\left[\begin{array}{l}
0 \\
1
\end{array}\right] \boldsymbol{\Phi}\right) \rho_{l} d s+\boldsymbol{\Lambda} \boldsymbol{q}=\boldsymbol{Q}_{q}
\end{aligned}
$$

Considering that the local coordinate centre is also the mass centre and its symmetry property about $w$ and $s$ axis, the following relationships hold:

$$
\begin{aligned}
& \int \rho_{l} d s=M_{f} \\
& \int s \rho_{l} d s=0 \\
& \int s^{2} \rho_{l} d s=I_{f} \\
& \int \eta^{2} \rho_{l} d s=\iint\left(\boldsymbol{\Phi}^{T} \boldsymbol{q}\right)\left(\boldsymbol{\Phi}^{T} \boldsymbol{q}\right) \rho_{l} d s=\boldsymbol{q}^{T}\left[\int \boldsymbol{\Phi} \rho_{l} \boldsymbol{\Phi}^{T} d s\right] \boldsymbol{q}=\boldsymbol{q}^{T} \mathbf{I q}=\boldsymbol{q}^{T} \boldsymbol{q} \\
& \int \eta \dot{\eta} \rho_{l} d s=\int\left(\boldsymbol{\Phi}^{T} \boldsymbol{q}\right)\left(\boldsymbol{\Phi}^{T} \dot{\boldsymbol{q}}\right) \rho_{l} d s=\boldsymbol{q}^{T} \dot{\boldsymbol{q}}
\end{aligned}
$$

where $I_{f}=M_{f}\left(L^{2}+H^{2}\right) / 12$ is the rotation inertia of the beam about mass centre.

If the mode function is chosen up to third-order, and by substituting the above relationships into Eqs. (2.68)-(2.70), the governing equations finally become:

$$
\begin{aligned}
& M \ddot{X}_{c R}+\dot{\theta}_{f}^{2} \sin \theta_{f}\left(\psi_{01} q_{1}+\psi_{02} q_{2}+\psi_{03} q_{3}\right)-2 \dot{\theta}_{f} \cos \theta_{f}\left(\psi_{01} \dot{q}_{1}+\psi_{02} \dot{q}_{2}+\psi_{03} \dot{q}_{3}\right) \\
& \quad-\ddot{\theta}_{f} \cos \theta_{f}\left(\psi_{01} q_{1}+\psi_{02} q_{2}+\psi_{03} q_{3}\right)-\sin \theta_{f}\left(\psi_{01} \ddot{q}_{1}+\psi_{02} \ddot{q}_{2}+\psi_{03} \ddot{q}_{3}\right)=Q_{X_{c R}} \\
& M \ddot{Y}_{c R}-\dot{\theta}_{f}^{2} \cos \theta_{f}\left(\psi_{01} q_{1}+\psi_{02} q_{2}+\psi_{03} q_{3}\right)-2 \dot{\theta}_{f} \sin \theta_{f}\left(\psi_{01} \dot{q}_{1}+\psi_{02} \dot{q}_{2}+\psi_{03} \dot{q}_{3}\right) \\
& \quad-\ddot{\theta}_{f} \sin \theta_{f}\left(\psi_{01} q_{1}+\psi_{02} q_{2}+\psi_{03} q_{3}\right)+\cos \theta_{f}\left(\psi_{01} \ddot{q}_{1}+\psi_{02} \ddot{q}_{2}+\psi_{03} \ddot{q}_{3}\right)+M g=Q_{Y_{c R}} \\
& -\left(\ddot{X}_{c R} \cos \theta_{f}+\ddot{Y}_{c R} \sin \theta_{f}\right)\left(\psi_{01} q_{1}+\psi_{02} q_{2}+\psi_{03} q_{3}\right)+I_{f} \ddot{\theta}_{f}+\ddot{\theta}_{f}\left(q_{1}{ }^{2}+q_{2}{ }^{2}+q_{3}{ }^{2}\right)+2 \dot{\theta}_{f}\left(\dot{q}_{1} q_{1}+\dot{q}_{2} q_{2}+\dot{q}_{3} q_{3}\right) \\
& \quad+\left(\psi_{11} \ddot{q}_{1}+\psi_{12} \ddot{q}_{2}+\psi_{13} \ddot{q}_{3}\right)=Q_{\theta} \\
& {\left[-\ddot{X}_{c R} \sin \theta_{f}+\ddot{Y}_{c R} \cos \theta_{f}\right] \psi_{01}+\psi_{11} \ddot{\theta}_{f}-\dot{\theta}_{f}^{2} q_{1}+\ddot{q}_{1}+\omega_{1}^{2} q_{1}=Q_{q_{1}}} \\
& {\left[-\ddot{X}_{c R} \sin \theta_{f}+\ddot{Y}_{c R} \cos \theta_{f}\right] \psi_{02}+\psi_{12} \ddot{\theta}_{f}-\dot{\theta}_{f}^{2} q_{2}+\ddot{q}_{2}+\omega_{2}^{2} q_{2}=Q_{q_{2}}} \\
& {\left[-\ddot{X}_{c R} \sin \theta_{f}+\ddot{Y}_{c R} \cos \theta_{f}\right] \psi_{03}+\psi_{13} \ddot{\theta}_{f}-\dot{\theta}_{f}^{2} q_{3}+\ddot{q}_{3}+\omega_{3}^{2} q_{3}=Q_{q_{3}}}
\end{aligned}
$$

where $Q_{q_{1}}, Q_{q_{2}}$ and $Q_{q_{3}}$ are the three components in Eq. (2.52), corresponding to the three modes. As can be seen, there are several non-linear terms in each of these equations, which represent the interaction effect between rigid-body motion and 
flexible deformation. This set of equations is then solved at each FSI iterations using Newmark method (Newmark, 1959) and Newton-Raphson methods. The detailed procedure of the solution process is given in the Appendix.

2.3.1.3. Symmetric flexible wedge. In this part, the case of a 2D wedge with flexible bottom vertically and freely dropping into water is investigated. The wedge model is shown in Fig. 2.3. Both of the bottoms are fixed at the junction point on the wedge top and free at the other ends. The bottoms are flexible whereas the other parts are treated as rigid body. Both of the two bottom beams have the same mass, which is defined as $M_{f}$, i.e.:

$$
M_{f A}=M_{f B}=M_{f}
$$

The symmetric property will make the dropping motion only occurs in the vertical direction and always perpendicular to the calm water surface, which means there would be no rotational motion during the dropping. Therefore, all the time derivatives of the angular variables are all zeros, i.e.:

$$
\dot{\theta}_{R}=\ddot{\theta}_{R}=\dot{\theta}_{f A}=\ddot{\theta}_{f A}=\dot{\theta}_{f B}=\ddot{\theta}_{f B}=0
$$

These angular conditions make all the angle-related terms in the general governing equations to be zeros. Moreover, the equation corresponding to angular motion (Eq. (2.63)) is not included since the angular variables are constants. As a result, the simplified governing equations for this case become:

$$
\begin{aligned}
& \sum_{i} \int_{f i}\left(\ddot{\boldsymbol{X}}_{c R}+\boldsymbol{R}_{f i} \ddot{\xi}_{i}\right) \rho_{l} d s+M_{R} \ddot{\boldsymbol{X}}_{c R}+\left(M_{R}+\sum_{i} M_{f i}\right) g\left[\begin{array}{l}
0 \\
1
\end{array}\right]=\boldsymbol{Q}_{X_{c R}} \\
& \int_{f i}\left(\ddot{\boldsymbol{X}}_{c R}^{T}+\ddot{\boldsymbol{\xi}}_{i}^{T} \boldsymbol{R}_{f i}^{T}\right)\left(\boldsymbol{R}_{f i}\left[\begin{array}{l}
0 \\
1
\end{array}\right] \boldsymbol{\Phi}_{i}\right) \rho_{l i} d s+\boldsymbol{\Lambda}_{i} \boldsymbol{q}_{i}=\boldsymbol{Q}_{q_{i}}
\end{aligned}
$$

To simplify further the forms of these equations, the following relations between $\theta_{f A}$ and $\theta_{f B}$, which are derived from the symmetric configuration of the wedge, are used to expand the vectors and matrices in the above equations, i.e.

$$
\begin{aligned}
& \sin \left(\theta_{f A}\right)=\sin \left(\pi-\theta_{f B}\right)=\sin \left(\theta_{f B}\right) \\
& \cos \left(\theta_{f A}\right)=\cos \left(\pi-\theta_{f B}\right)=-\cos \left(\theta_{f B}\right)
\end{aligned}
$$

Moreover, the vertical motion constraint also means the wedge should be motionless in the horizontal direction, i.e.:

$$
X_{c R}=\dot{X}_{c R}=\ddot{X}_{c R}=0
$$

As the wedge is symmetric about the $y_{R}$ axis and the entry speed is perpendicular to the free surface, the flexible deformation of the two bottom beams will have the same absolute amount but opposite direction when measured in their corresponding local $x_{f i}-y_{f i}$ coordinate system. Therefore, if the mode functions for the left beam $\boldsymbol{\Phi}_{A}$ are chosen to be the opposite ones of the right beam $\boldsymbol{\Phi}_{B}$, i.e.:

$$
\boldsymbol{\Phi}_{B}=-\boldsymbol{\Phi}_{A}=\boldsymbol{\Phi}
$$

where $\boldsymbol{\Phi}$ is the is the vector of the mode function defined by Eq. (2.30), then the generalised coordinates $\boldsymbol{q}_{i}$ would be

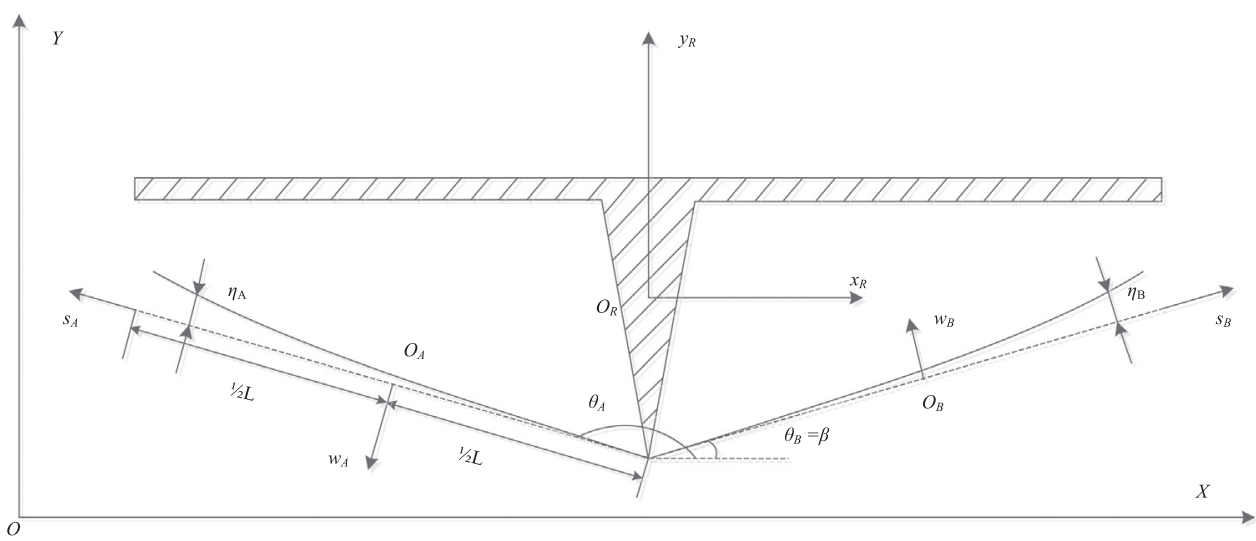

Fig. 2.3. sketch of the wedge with flexible bottom. 
identical for both sides, that is,

$$
\boldsymbol{q}_{A}=\boldsymbol{q}_{B}
$$

Substituting Eqs. (2.89) and (2.90) into Eq. (2.52) (for the calculation of $\boldsymbol{Q}_{q_{i}}$ ), and considering the water pressure ( - pn) pointing in the same and opposite direction of $w_{i}$ for Beam A $\left(\boldsymbol{e}_{A w}\right)$ and Beam B $\left(\boldsymbol{e}_{B w}\right)$, respectively (which means (pn) $\boldsymbol{e}_{A w}$ and (pn) $\boldsymbol{e}_{B w}$ would be of the opposite sign), then the value of the generalized force $\boldsymbol{Q}_{q_{i}}$ for both of the two beams would be the same, i.e.:

$$
\boldsymbol{Q}_{q_{A}}=\boldsymbol{Q}_{q_{B}}
$$

After substituting the above relations, the final governing equations for the flexible wedge dropping problem are:

$$
\begin{aligned}
& \left(2 M_{f}+M_{R}\right) \ddot{Y}_{c R}+2 \cos \theta\left(\psi_{01} \ddot{q}_{1}+\psi_{02} \ddot{q}_{2}+\psi_{03} \ddot{q}_{3}\right)+\left(2 M_{f}+M_{R}\right) g=Q_{Y_{c R}} \\
& 2 \ddot{Y}_{c R} \cos \theta \psi_{01}+2 \ddot{q}_{1}+2 \omega_{1}^{2} q_{1}=2 Q_{q_{1}} \\
& 2 \ddot{Y}_{c R} \cos \theta \psi_{02}+2 \ddot{q}_{2}+2 \omega_{2}^{2} q_{2}=2 Q_{q_{2}} \\
& 2 \ddot{Y}_{c R} \cos \theta \psi_{03}+2 \ddot{q}_{3}+2 \omega_{3}^{2} q_{3}=2 Q_{q_{3}}
\end{aligned}
$$

where the subscript $i(i=A, B)$, which is used for indicating different beams in $\boldsymbol{q}_{i}, \boldsymbol{\psi}_{i 0}, \boldsymbol{\psi}_{i 1}$ and $\boldsymbol{Q}_{q_{i}}$, is omitted based on the equivalent conditions aforementioned. What is worth mentioning is that the angle $\theta$ and mode function vector $\boldsymbol{\psi}_{0}$ are chosen to be the value of beam B, although the structure of these equations would be the same if they are chosen from beam A..As before, this set of equations is also solved by coupled Newmark and Newton-Raphson methods. The detailed formulation is given in Appendix.

\section{Fluid solver-Modified MPS method}

In this section, a brief illustration about the modified MPS methodology would be given. More details could be found in (Sun et al., 2014, 2015).

\subsection{Governing equations}

The problems investigated in this paper are all marine related violent and rapid changing physical processes, which mean the viscosity effect is quite small. In the Lagrangian frame, the incompressible and inviscid Navier-Stokes equations are given by

$$
\begin{gathered}
\frac{D \boldsymbol{u}}{D t}=\boldsymbol{g}-\frac{\nabla p}{\rho} \\
\nabla \cdot \boldsymbol{u}=0
\end{gathered}
$$

where $\boldsymbol{u}, p$ and $\rho$ are the fluid velocity, pressure and density respectively, and $\mathbf{g}$ is the vector pointing to the gravity direction, i.e. $\mathbf{g}=[0,-g]$. In the Lagrangian frame, there is no convection term in the acceleration of the momentum equation (i.e., the left hand side of the first equation in Eq. (3.1)). The position of each particle is advanced based on its own physical velocity i.e. $\frac{D \boldsymbol{r}}{D t}=\boldsymbol{u}(\boldsymbol{r}$ is the position vector of a particle). This process is illustrated in Section 3.2 below.

\subsection{Enforcing incompressibility-Projection method}

As a typical approach for the incompressible fluid computation, the two-step projection method, which is introduced by Chorin (Chorin, 1967), is adopted here to decouple the velocity and pressure calculation.

The first step is to calculate the intermediate velocity without considering pressure, and then move the particles to the intermediate location according to this velocity:

$$
\left\{\begin{array}{l}
\boldsymbol{u}_{*}=\boldsymbol{u}_{n}+\Delta t \mathbf{g} \\
\boldsymbol{r}_{*}=\boldsymbol{r}_{n}+\Delta t \boldsymbol{u}_{*}
\end{array}\right.
$$

A pressure Poisson equation is then derived as follows to solve the pressure field:

$$
\nabla^{2} p_{n+1}=\frac{\rho \nabla \cdot \boldsymbol{u}_{*}}{\Delta t}+\alpha \rho \frac{n_{0}-n_{n}}{n_{0} \Delta t^{2}}
$$

Here, the term $n_{0}$ and $n_{n}$ are called "particle density". The subscripts 0 and $n$ indicate the initial and the last time step states, respectively. They are proportional to the physical density. Their definitions will be given in Section 3.3. 
The coefficient $\alpha$ in Poisson equation (Eq. (3.3)) is chosen in the following way (Sun et al., 2014):

$$
\alpha= \begin{cases}\left|\frac{n_{0}-n_{n}}{n_{0}}\right|+\Delta t\left|\nabla \cdot \boldsymbol{u}_{n}\right| & \text { if }\left(n_{0}-n_{n}\right) \nabla \cdot \boldsymbol{u}_{n} \geq 0 \\ \left|\frac{n_{0}-n_{n}}{n_{0}}\right| & \text { if }\left(n_{0}-n_{n}\right) \nabla \cdot \boldsymbol{u}_{n} \leq 0\end{cases}
$$

which means no artificial term appears in the process of the determination of $\alpha$.

The condition $\left(n_{0}-n_{n}\right) \nabla \cdot \boldsymbol{u}_{n} \geq 0$ means the fluid is compressed i.e. $\left(n_{0}-n_{n}\right) \leq 0$ (or expanded i.e. $\left.\left(n_{0}-n_{n}\right) \geq 0\right)$ in the last time step $(t=n \Delta t)$ and will be further compressed according to the motion trend of particles i.e. $\nabla \cdot \boldsymbol{u}_{n} \leq 0$ (or expanded i.e. $\left.\nabla \cdot \boldsymbol{u}_{n} \geq 0\right)$. Under this situation, an additional term $\left(\Delta t\left|\nabla \cdot \boldsymbol{u}_{n}\right|\right)$ is added into the coefficient to help to control this further compression (or expanding).

After obtaining the pressure, the velocity and location are then updated as:

$$
\left\{\begin{array}{l}
\boldsymbol{u}_{n+1}=\boldsymbol{u}_{*}-\Delta t \cdot \frac{\nabla p_{n+1}}{\rho_{0}}(a) \\
\boldsymbol{r}_{n+1}=\boldsymbol{r}_{n}+\Delta t \cdot \boldsymbol{u}_{n+1}(b)
\end{array}\right.
$$

\subsection{Particle interaction model}

The gradient and Laplacian operators are discretized by a weighted average approach:

$$
\begin{aligned}
& \nabla u\left(\boldsymbol{x}_{i}\right)=\frac{d}{n_{0}} \sum_{j \neq i}^{M} \frac{u\left(\boldsymbol{x}_{j}\right)-u\left(\boldsymbol{x}_{i}\right)}{r_{i j}^{2}}\left(\boldsymbol{x}_{j}-\boldsymbol{x}_{i}\right) w\left(r_{i j}\right) \\
& \nabla^{2} u\left(\boldsymbol{x}_{i}\right)=\frac{2 d}{n_{0} \lambda} \sum_{j \neq i}^{M}\left[u\left(\boldsymbol{x}_{j}\right)-u\left(\boldsymbol{x}_{i}\right)\right] w\left(r_{i j}\right)
\end{aligned}
$$

where $d$ is the number of space dimension, $M$ is particles number in the support domain, $w\left(r_{i j}\right)$ is the weight function and $\lambda$ is a parameter related to $w\left(r_{i j}\right)$ by:

$$
\begin{aligned}
& w\left(r_{i j}\right)=\left\{\begin{array}{rl}
\frac{r_{e}}{r_{i j}}-1 & 0 \leq r_{i j} \leq r_{e} \\
0 & r_{i j} \geq r_{e}
\end{array}\right. \\
& \lambda=\frac{\sum_{j \neq i}^{M} w\left(r_{i j}\right) r_{i j}{ }^{2}}{\sum_{j \neq i}^{M} w\left(r_{i j}\right)}
\end{aligned}
$$

where $r_{e}$ is the radius of local support domain.

The particle density is also defined based on the weight function as follows:

$$
n=\sum_{j \neq i}^{M} w\left(r_{i j}\right)
$$

After applying these discretizing models to the pressure Poisson equation, a linear system is then obtained as:

$$
\boldsymbol{A} \cdot \boldsymbol{P}=\boldsymbol{B}
$$

where $\boldsymbol{A}$ and $\boldsymbol{B}$ are the coefficient matrix and the right hand side vector, respectively. $\boldsymbol{P}=\left[p_{1}, p_{2}, \ldots, p_{n},\right]^{T}$ is the unknown discretized node pressure vector. The system is then solved by Generalized Minimum Residual (GMRES) method.

\subsection{Boundary conditions for Poisson equation}

a) Pressure Neumann condition on solid boundary

In the proposed scheme, the following Neumann condition (Eq. (3.12)) is applied on the inner most layer of solid boundary, instead of Poisson equation (Eq. (3.3)) as in standard MPS. The gradient of pressure is calculated between the current boundary particle and the nearest fluid particle.

$$
\boldsymbol{n} \cdot \nabla p_{n+1}=\rho\left(\boldsymbol{n} \cdot \mathbf{g}-\boldsymbol{n} \cdot \dot{\boldsymbol{U}}_{n+1}\right) \cong \rho\left(\boldsymbol{n} \cdot \mathbf{g}-\boldsymbol{n} \cdot \dot{\boldsymbol{U}}_{n}\right)
$$

where $\dot{U}$ is the acceleration of the boundary. In the case that the motion of the boundary is determined by the pressure of the surrounding fluid, the acceleration of next time step $\dot{\boldsymbol{U}}_{n+1}$ is unknown since the pressure has not been solved yet. As an approximation, the value of the last time step (or the last iteration when iterative process is involved in the fluid structure interaction) for $\dot{\boldsymbol{U}}_{n}$ is adopted instead.

b) Laplacian operator compensation near solid boundary

For the fluid particles which are close to the solid boundary, the Laplacian operator is modified to be consistent with the Newman condition. As shown in Fig. 3.1, if the virtual particle, which is along the local normal direction and away from 


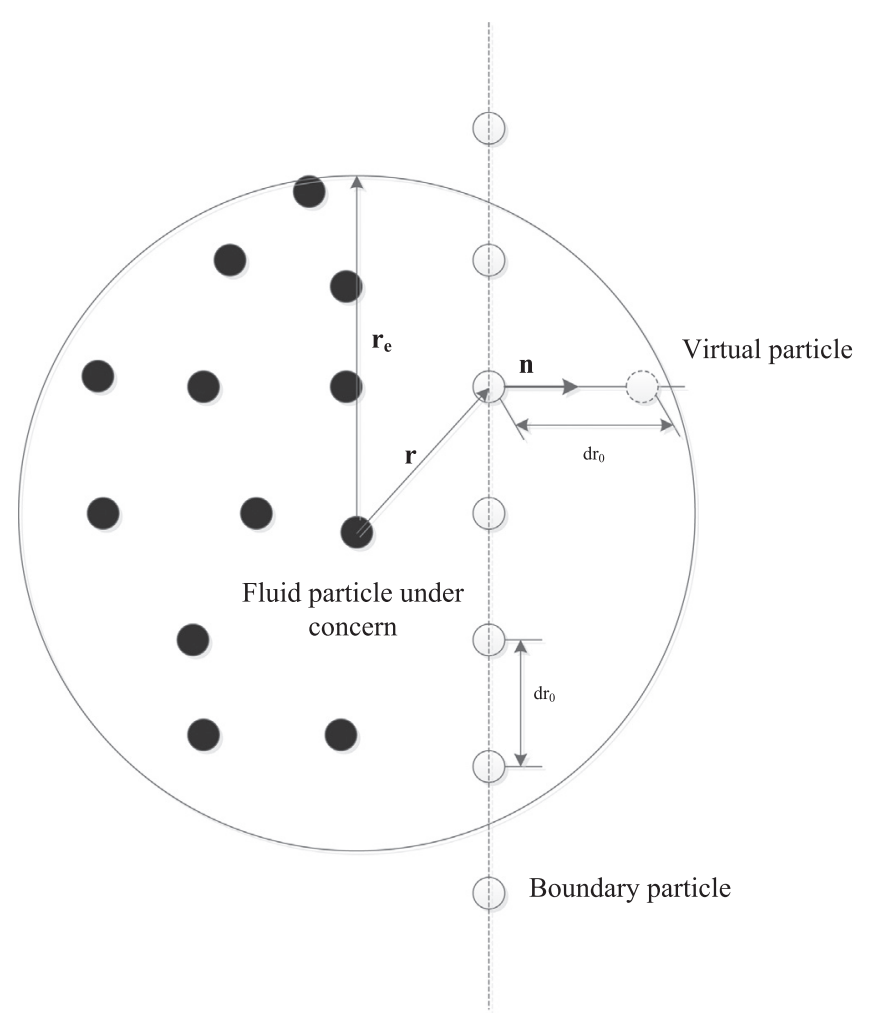

Fig. 3.1. Demonstration of new pressure boundary condition.

solid boundary with the initial distancedr ${ }_{0}$, is within the support domain of the fluid particle, this virtual particle is also included in the discretization of Laplacian operator. The pressure (which is unknown) of this virtual particle is then represented by the corresponding solid particle based on Eq. (3.12), i.e.

$$
p_{V}=p_{S}+\rho(\boldsymbol{n} \cdot \mathbf{g}-\boldsymbol{n} \cdot \dot{\boldsymbol{U}}) d r_{0}
$$

where, $p_{V}$ is the pressure of virtual particle, and $p_{S}$ is the pressure of the corresponding solid particle.

c) Intermediate velocity of solid boundary particles

The choice of intermediate velocity $\boldsymbol{u}_{*}$ on solid boundary will affect the accuracy of pressure, since the divergence of intermediate velocity is the source term of the pressure Poisson equation. As suggested by (Brown et al., 2001), the choice of $\boldsymbol{u}_{*}$ should guarantee that, at $(n+1)^{\text {th }}$ time step, the fluid velocity on boundary $\left(\left.\boldsymbol{u}_{n+1}\right|_{\partial \Omega}\right)$ should be equal to the solid body velocity on the fluid-solid interface $\left(\boldsymbol{u}_{b n+1}\right)$.

Finally, by using Eqs. (3.5a) and (3.12), the intermediate velocities of the boundary particles are chosen as:

$$
\begin{aligned}
\frac{\partial \boldsymbol{u}_{\boldsymbol{b}_{*}}}{\partial n} & =\boldsymbol{n} \cdot \boldsymbol{u}_{\boldsymbol{b} n+1}+\Delta t\left(\boldsymbol{n} \cdot \mathbf{g}-\boldsymbol{n} \cdot \dot{\boldsymbol{U}}_{n}\right) \\
\frac{\partial \boldsymbol{u}_{\boldsymbol{b}_{*}}}{\partial \tau} & =\boldsymbol{\tau} \cdot \boldsymbol{u}_{\boldsymbol{b}_{n+1}}+\frac{\Delta t}{\rho_{0}} \frac{\partial p_{n}}{\partial \tau}
\end{aligned}
$$

The derivative terms are calculated by simple finite difference approach between its neighbor solid particles.

As pointed out in (Brown et al., 2001), using the above intermediate velocity boundary condition Eq. (3.14), the time accuracy for both the velocity and the pressure (Eqs. (3.2)-(3.5)) are of second-order.

a) Free surface particle identification

A simplified version of the method used by Koh et al (Koh et al., 2012) is adopted. If the "circle" is completely covered by its neighbors, then it is recognized as an inner fluid particle, otherwise it is a free surface particle. The circle is discretized by 360 points which locate evenly along the circle. If all these points are covered, the circle is then regarded as being covered. For example, in Fig. 3.2, particle A is recognized as free surface, because the yellow points on its "circle" are not covered by its neighbors. In contract, particle B is identified as inner fluid particle. 


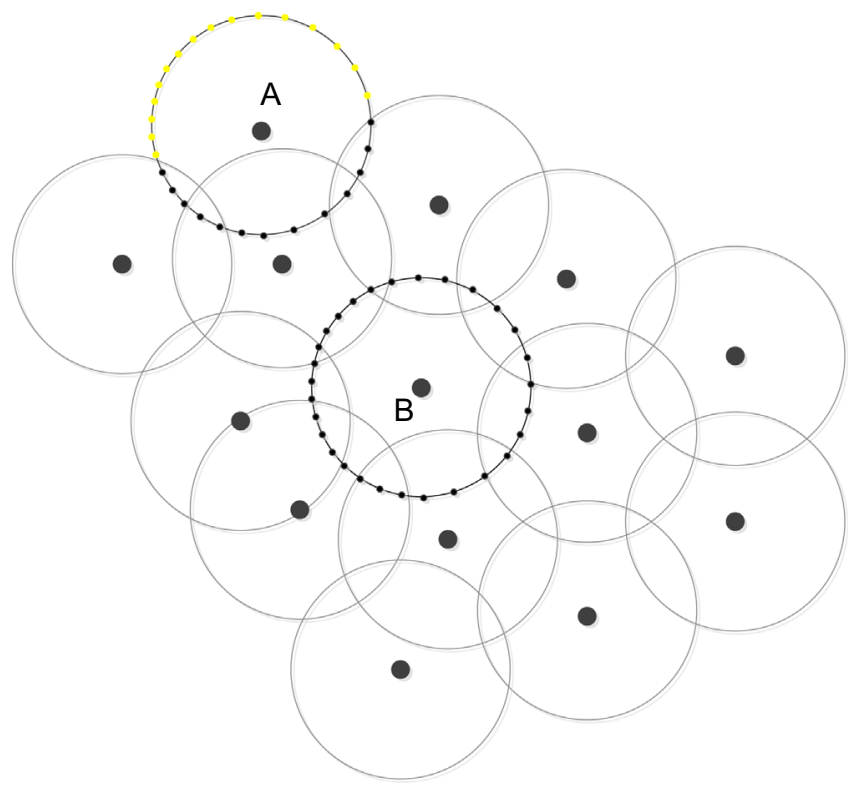

Fig. 3.2. Demonstration of free surface particle recognition method.

\subsection{Particle shifting and collision handling}

In order to alleviate the disorder of particle distribution, after each time step, the positions of particles are slightly shifted. Actually, this technique could be regarded as a re-meshing procedure. Moreover, because the amount of shifting is very small, not mapping the value onto the new positions will not corrupt the results.

The amount of shifting is chosen as:

$$
\delta \boldsymbol{r}_{i}=\sum_{j \neq i} \frac{r_{0-}\left|\boldsymbol{r}_{i j}\right|}{2} \cdot \frac{\boldsymbol{r}_{i-} \boldsymbol{r}_{j}}{\left|\boldsymbol{r}_{i j}\right|}, \quad \text { when }\left|\boldsymbol{r}_{i j}\right| \leq \bar{r}_{0}
$$

where $\overline{\mathrm{r}}_{0}$ is normally set to be $99 \%$ of the initial particle distance, i.e. $\overline{\mathrm{r}}_{0}=0.99 \times \mathrm{dr}_{0}$. Furthermore, before the calculation at each time step, we apply the following velocity manipulation for each fluid particle to avoid potential collisions:

$$
\delta \boldsymbol{v}_{i}=\sum_{j \neq i}-\epsilon\left(\boldsymbol{r}_{i j}\right) \boldsymbol{v}_{t i j}, \quad \text { when }\left|\boldsymbol{r}_{i j}-\boldsymbol{v}_{i i j} \Delta t\right| \leq r_{\text {min }}
$$

where $\boldsymbol{v}_{\tau i j}$ is the tangential relative velocity between particles $i$ and $j$, and $r_{\min }$ is the threshold to activate the scheme. It is selected as roughly $30 \%$ of the initial particle distance in this study. Parameter $\epsilon$ depends on the property of particlej. If particlej is a fluid particle, $\epsilon$ is equal to 0.5 , otherwise, if it is a solid boundary particle, $\epsilon$ is equal to 1.0. This setting is to make sure that the solid particles velocity involved will not be affected, while the relative velocity between its neighbour fluid particles will still be set to zero.

\subsection{Neighbour particle searching strategy}

As shown in Fig. 3.3, in order to discretizing the Laplacian and gradient operators, the particles which are located within the local support domain need to be found and kept updated when the particle relative locations change. The most commonly used method, the "cell-link" method, is as follows: The whole computation domain is first divided into regular grids (cells) of the size of at least the radius of support domain for Laplacian (4.0 times of initial particle distance $4.0 \times \mathrm{dr}_{0}$, which is larger than the supporting domain for gradient, i.e. 2.1 times of the initial particle distance). Then, the searching for each particle is conducted within its nearest "cells" (9 cells in 2D, 27 cells in 3D).

In this study, the size of the cell is reduced to the initial particle distance (i.e.dr $\mathrm{r}_{0}$ ). This modification makes the new searching area (area covered by red line in Fig. 3.3) becomes about 4/9 of the original one (area covered by green line in Fig. 3.3), which greatly improves the efficiency. Moreover, the non-redundant particle searching strategy (Crespo, 2008) is also employed here. By using this technique, only the cells with higher indexes are required to be searched, which means the searching area is further reduced into the one covered by blue color if the cells are indexed vertically from bottom to top. Finally, only about $2.5 / 9$ of the traditional searching area is required by using the proposed approach. More details could be found in (Sun et al., 2015). 


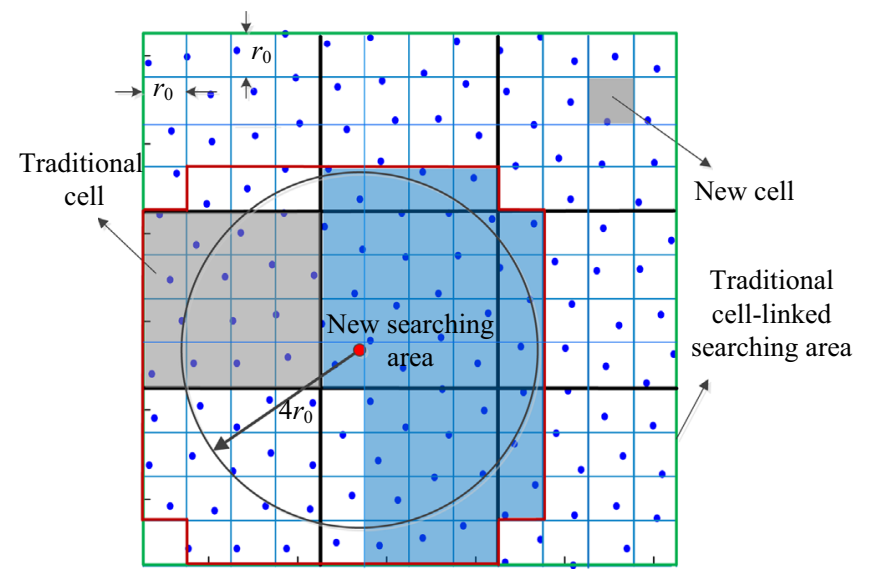

Fig. 3.3. Demonstration of the new neighbour particle searching strategy.

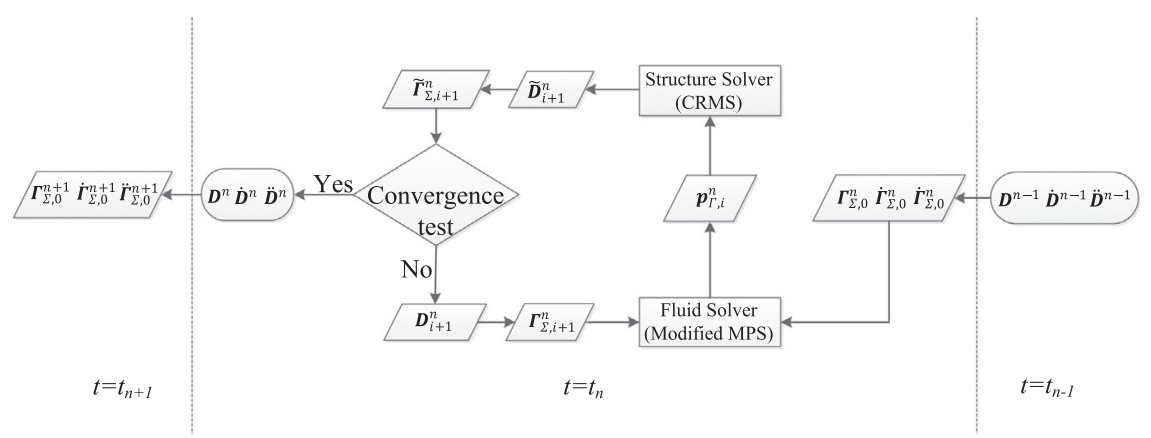

Fig. 4.1. Flow chart of the iterative process of fluid structure interaction.

\section{Fluid structure interaction strategy}

The interaction between the structure and water is computed in an iterative way. The Gauss-Seidel method with Aitken relaxation approach is adopted in this study.

We suppose that all the fluid and structure variables are known at $t=t_{n-1}$. Then, the detailed process of interaction is illustrated as follows:

1) Predict the position, velocity and acceleration of the points on fluid structure interface $\boldsymbol{\Gamma}_{\Sigma, 0}^{n}, \dot{\boldsymbol{\Gamma}}_{\Sigma, 0}^{n}$ and $\ddot{\boldsymbol{\Gamma}}_{\Sigma, 0}^{n}$ at $t=t_{n}$ using the structure kinetic values at $t=t_{n-1}$. i.e., $\boldsymbol{D}^{n-1}, \dot{\boldsymbol{D}}^{n-1}$ and $\ddot{\boldsymbol{D}}^{n-1}$ (Eqs. (2.4) and (2.10) in Section 2.1)

2) Based on the updated kinetic information of interface, calculate the fluid motion at $t=t_{n}$, by the modified MPS method. Then, obtain the new pressure $p_{\Sigma, i}^{n}$ applied on the interface for $i^{\text {th }}$ iteration at $t=t_{n}$.

3) Use the new fluid pressure $p_{\Sigma, i}^{n}$ to update the structure position, velocity and acceleration by the structural model for $t=t_{n}$, i.e. , $\tilde{\boldsymbol{D}}_{i+1}^{n}, \dot{\tilde{\boldsymbol{D}}}_{i+1}^{n}$ and $\tilde{\tilde{\boldsymbol{D}}}_{i+1}^{n}$. Then, find the corresponding kinetic values $\tilde{\boldsymbol{\Gamma}}_{\Sigma, i+1}^{n} \dot{\tilde{\boldsymbol{\Gamma}}}_{\Sigma, i+1}^{n}$ and $\tilde{\tilde{\boldsymbol{\Gamma}}}_{\Sigma, i+1}^{n}$ of the points on the interface (by Eqs. (2.4) and (2.10) in Section 2.1).

4) Check the difference between $\tilde{\boldsymbol{\Gamma}}_{\Sigma, i+1}^{n}$ and $\boldsymbol{\Gamma}_{\Sigma, i}^{n}$. If the convergence condition

$$
\left|\tilde{\boldsymbol{\Gamma}}_{\Sigma, i+1}^{n}-\boldsymbol{\Gamma}_{\Sigma, i}^{n}\right| \leq \epsilon
$$

is satisfied, then go to step (1) to continue the computation for the next time step $\left(t=t_{n+1}\right)$.

Otherwise, correct the structure position $\boldsymbol{D}_{i+1}^{n}$ for $(i+1)^{\text {th }}$ iteration using Eq. (4.2):

$$
\boldsymbol{D}_{i+1}^{n}=\chi_{i} \tilde{\mathbf{D}}_{i+1}^{n}+\left(1-\chi_{i}\right) \boldsymbol{D}_{i}^{n}
$$

and update the velocity $\dot{\boldsymbol{D}}_{i+1}^{n}$ and acceleration $\ddot{\boldsymbol{D}}_{i+1}^{n}$ by Newmark method (see Appendix). The corresponding interface variables $\boldsymbol{\Gamma}_{\Sigma, i+1}^{n}, \dot{\boldsymbol{\Gamma}}_{\Sigma, i+1}^{n}$ and $\ddot{\boldsymbol{\Gamma}}_{\Sigma, i+1}^{n}$ are then calculated according to $\boldsymbol{D}_{i+1}^{n}, \dot{\boldsymbol{D}}_{i+1}^{n}$ and $\ddot{\boldsymbol{D}}_{i+1}^{n}$.

Using these corrected interface information, conduct $(i+1)^{\text {th }}$ iteration by going back to step (2). 


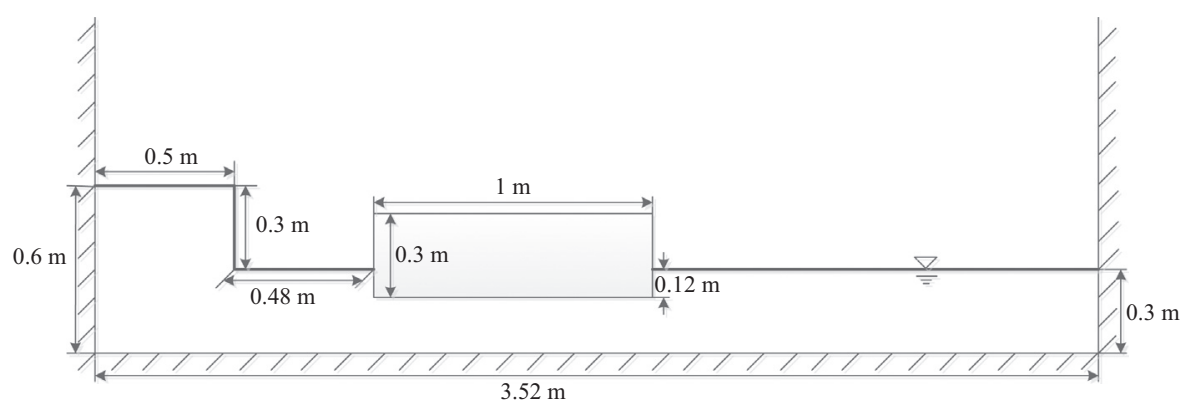

Fig. 5.1. Initial configuration of free floating beam case.

Here $\chi_{i}$ is the Aitken relaxation factor, and its value is calculated by the following equation:

$$
\chi_{i}=-\chi_{i-1} \frac{\Delta \boldsymbol{\Gamma}_{\Sigma, i+1}^{n}{ }^{T}\left(\Delta \boldsymbol{\Gamma}_{\Sigma, i+1}^{n}-\Delta \boldsymbol{\Gamma}_{\Sigma, i}^{n}\right)}{\left(\Delta \boldsymbol{\Gamma}_{\Sigma, i+1}^{n}-\Delta \boldsymbol{\Gamma}_{\Sigma, i}^{n}\right)^{T}\left(\Delta \boldsymbol{\Gamma}_{\Sigma, i+1}^{n}-\Delta \boldsymbol{\Gamma}_{\Sigma, i}^{n}\right)},
$$

in which $\Delta \boldsymbol{\Gamma}_{\Sigma, j}^{n}=\tilde{\boldsymbol{\Gamma}}_{\Sigma, j}^{n}-\boldsymbol{\Gamma}_{\Sigma, j-1}^{n}$.

This procedure is summarized in Fig. 4.1:

\section{Results and discussion}

\subsection{Free floating beam}

The testing case of a break-dam hitting floating flexible beam is calculated. The initial configuration is given in Fig. 5.1. The simulation starts from the equilibrium state in which the beam gravity is balanced out by the water buoyancy.

Two cases with different flexibility have been simulated. The conditions are as follows:

Case 1 (High flexibility):

Bending stiffness $E J=4.5 \mathrm{Nm}^{2}$ density $\rho=400 \mathrm{~kg} / \mathrm{m} 3$ and the first three nature circular frequencies are: $\omega_{1}=4.3325, \omega_{2}=11.9429, \omega_{1}=23.4128$

Case 2 (Low flexibility):

Bending stiffness $E J=4.5 \times 10^{2} \mathrm{Nm}^{2}$; density $\rho=400 \mathrm{~kg} / \mathrm{m}^{3}$; and the first three nature circular frequencies are: $\omega_{1}=43.3249, \omega_{2}=119.4288, \omega_{3}=234.1281$

The fluid domain is discretised by particles with an initial distance of $0.02 \mathrm{~m}$. As a consequence, the total fluid particles number is 2643 . The time interval is determined by the CFL condition with an upper limit of $0.001 \mathrm{~s}$.

The simulations are conducted on a computer with Intel(R) Core(TM) i5-2400 (duo 3.1 GHz) CPU, RAM 4.0 GB. The physical duration is 5 seconds (about 5000 time steps). Each of the two cases requires approximately 2 CPU hours' time with the above computer.

The comparison of high and low flexibility cases are given in Figs. 5.2 and 5.3. For the rigid-body motion part, the effect of flexibility is accumulated to a remarkable level during the simulation period, especially for the translational variables (Fig. 5.2(a) and (b)). This shows that for violent water flexible structure interaction problem, the coupling term should be taken into account to get accurate overall trajectory. For the elastic motion part, as is shown in Fig. 5.3, the values for low flexibility are much smaller than that of high flexibility, as expected. Moreover, the periods of these curves are basically consistent with the corresponding dominant nature frequency.

The pressure contour of the fluid field and the deformation of the beam for the two cases are depicted in Fig. 5.4. As is shown, for both cases there is no singular point in the fluid field and the pressure field is quite smooth. The beam motion is physically reasonable as well.

The structure solver turns out to be quite efficient. For most of the time, only one time iteration is required for the Newton-Raphson method to reach the convergence criteria of $10^{-5}$, and the computation time for structure is negligible.

The average iteration times required for the FSI coupling is 2.8 for an accuracy of $10^{-5}$.

\subsection{Wedge with flexible bottom}

The results of the flexible wedge dropping case are discussed in this section. Fig. 5.5 shows the initial configuration of the problem. The numerical results are also compared with the experimental data provided in (Panciroli, 2013). Considering 
(a)

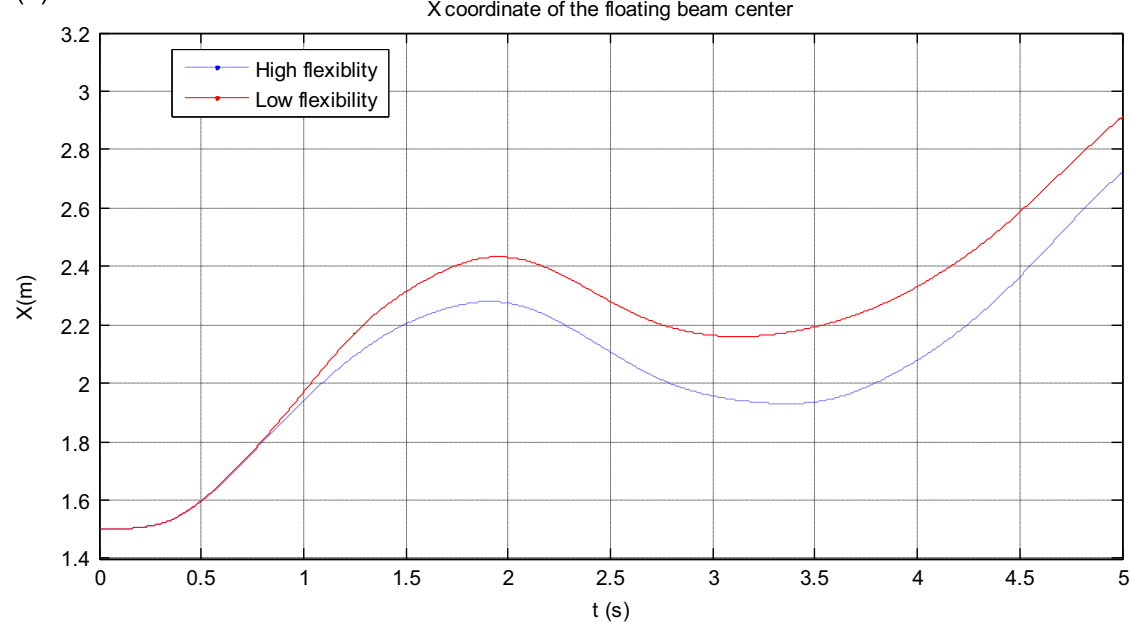

(b)

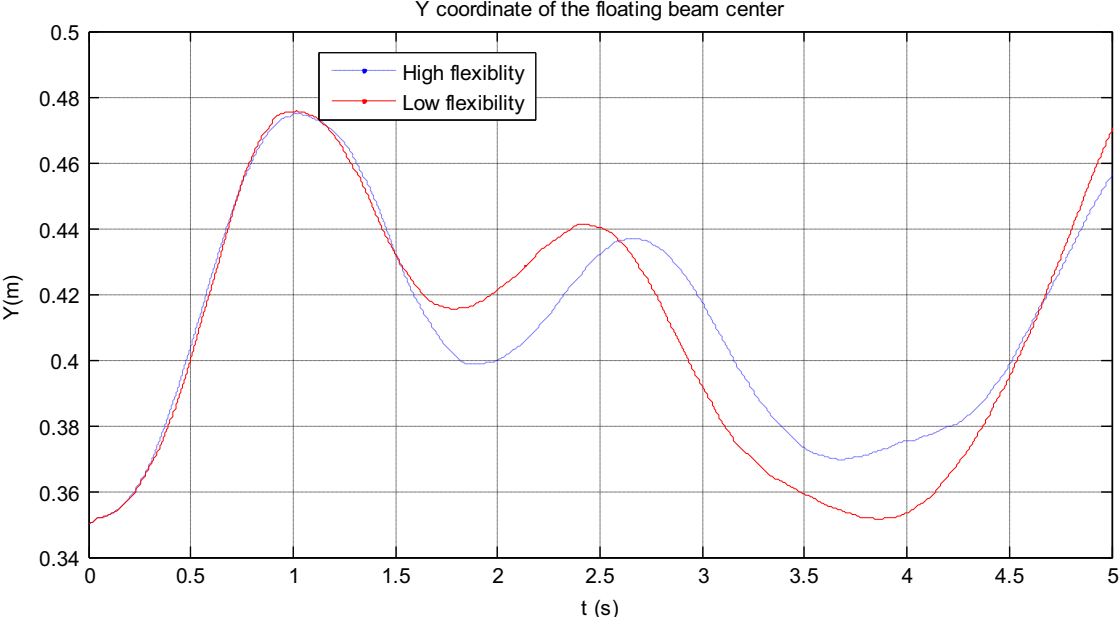

(c)

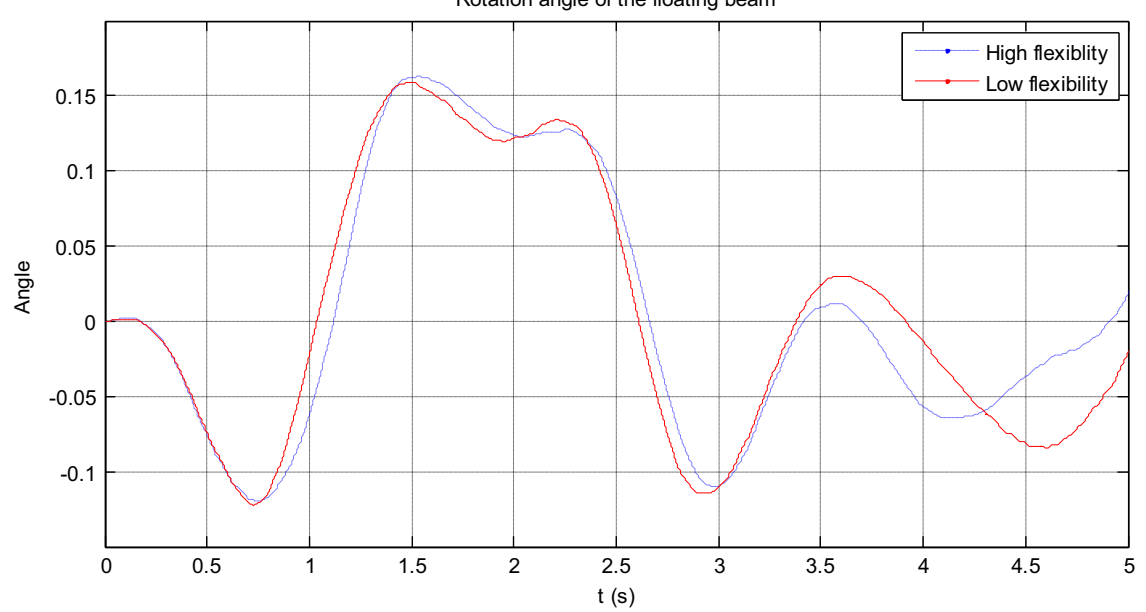

Fig. 5.2. The comparison of the rigid-body motion time history for different flexibilities: (a) X coordinate of mass centre; (b) $\mathrm{Y}$ coordinate of mass centre; (c) Rotation angle round mass centre. 

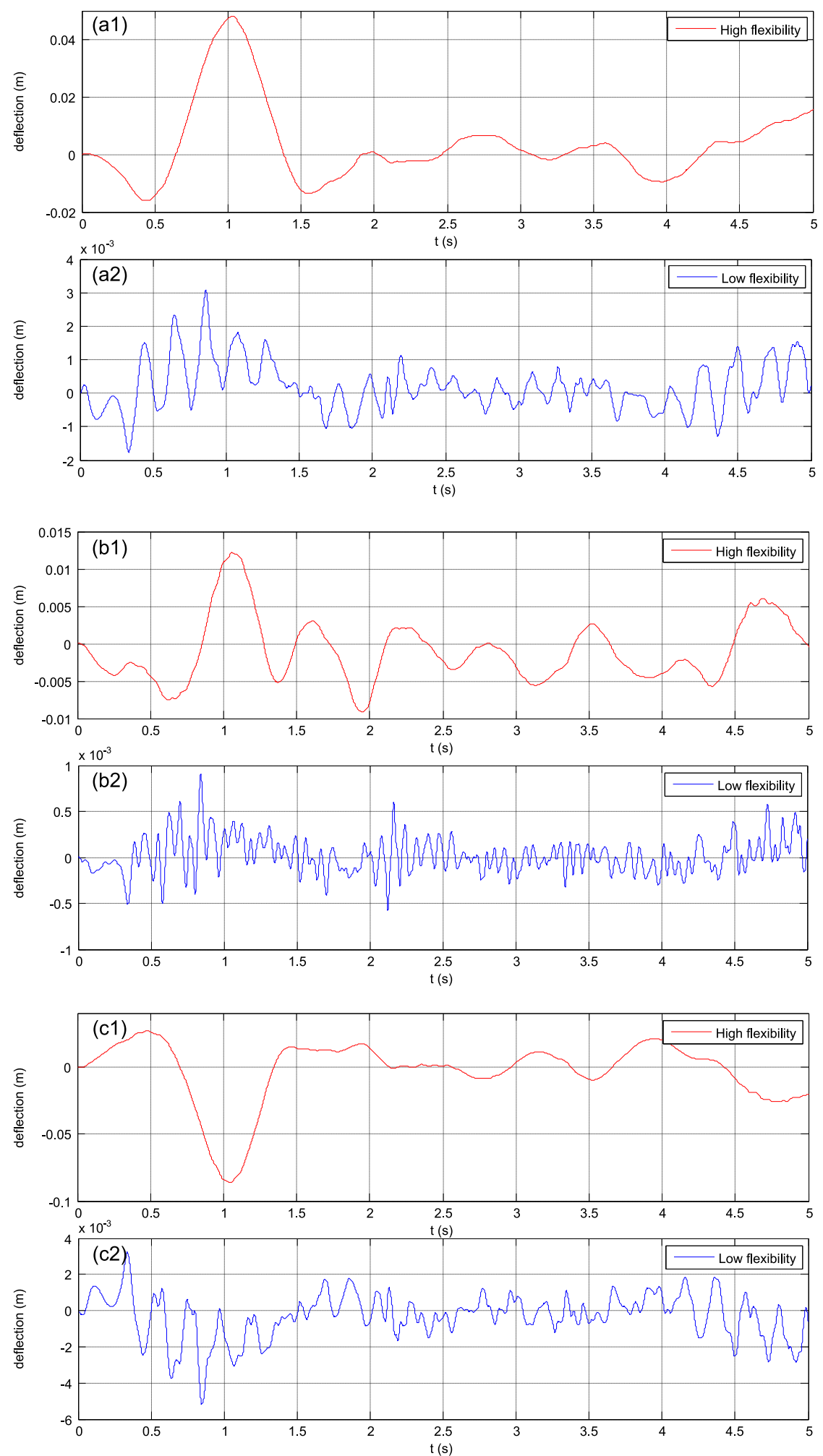

Fig. 5.3. The comparison of deflection time history for different flexibilities at different positions: (a1) \& (a2) middle of the beam; (b1) \& (b2) far-end quarter of the beam; (c1) \& (c2) far-end of the beam. 
High flexibility
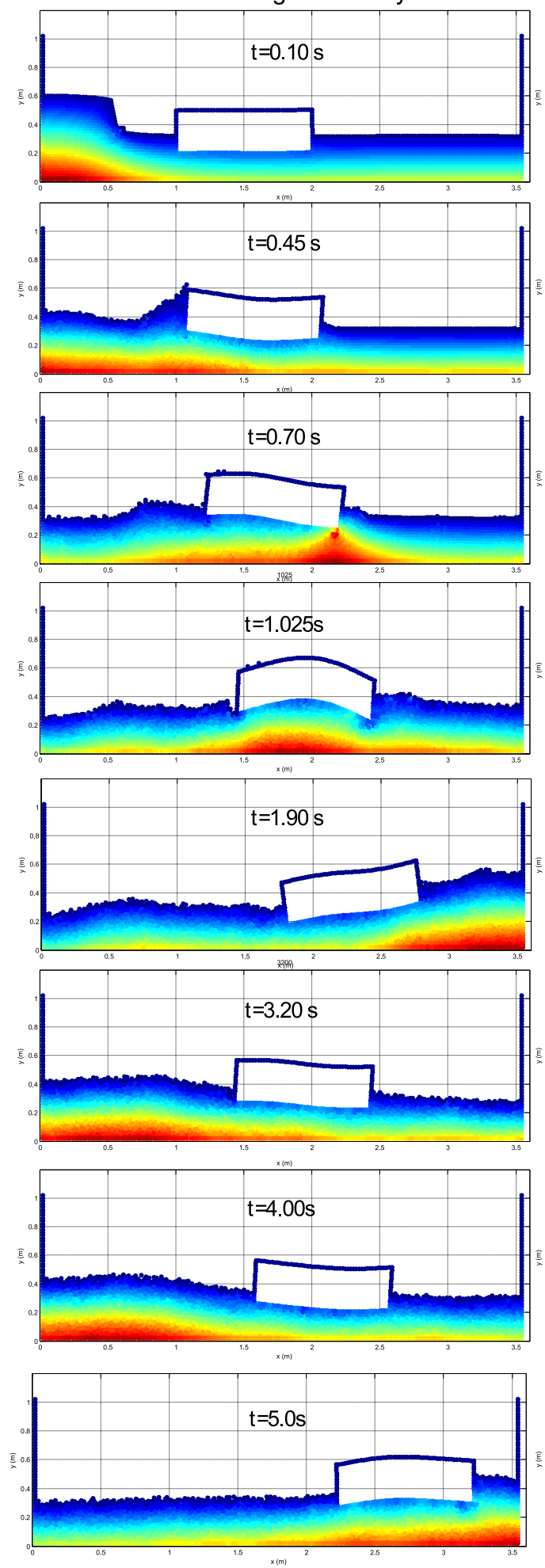

Low flexibility
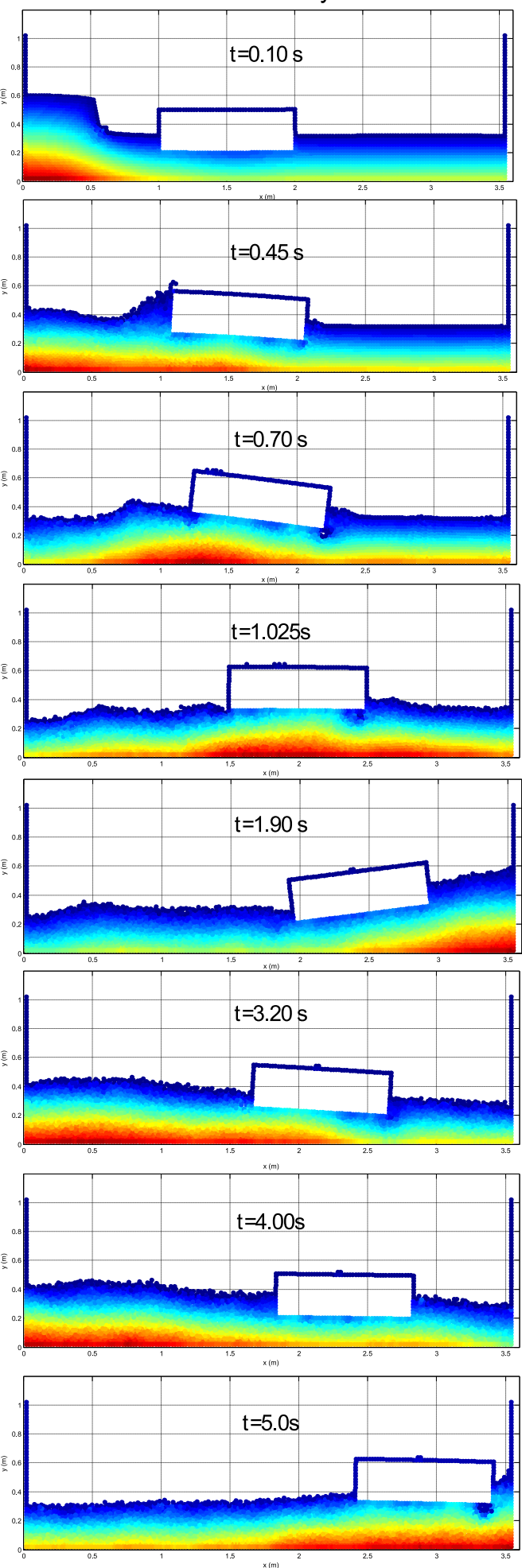

Fig. 5.4. Pressure contour comparison of different flexibility. 


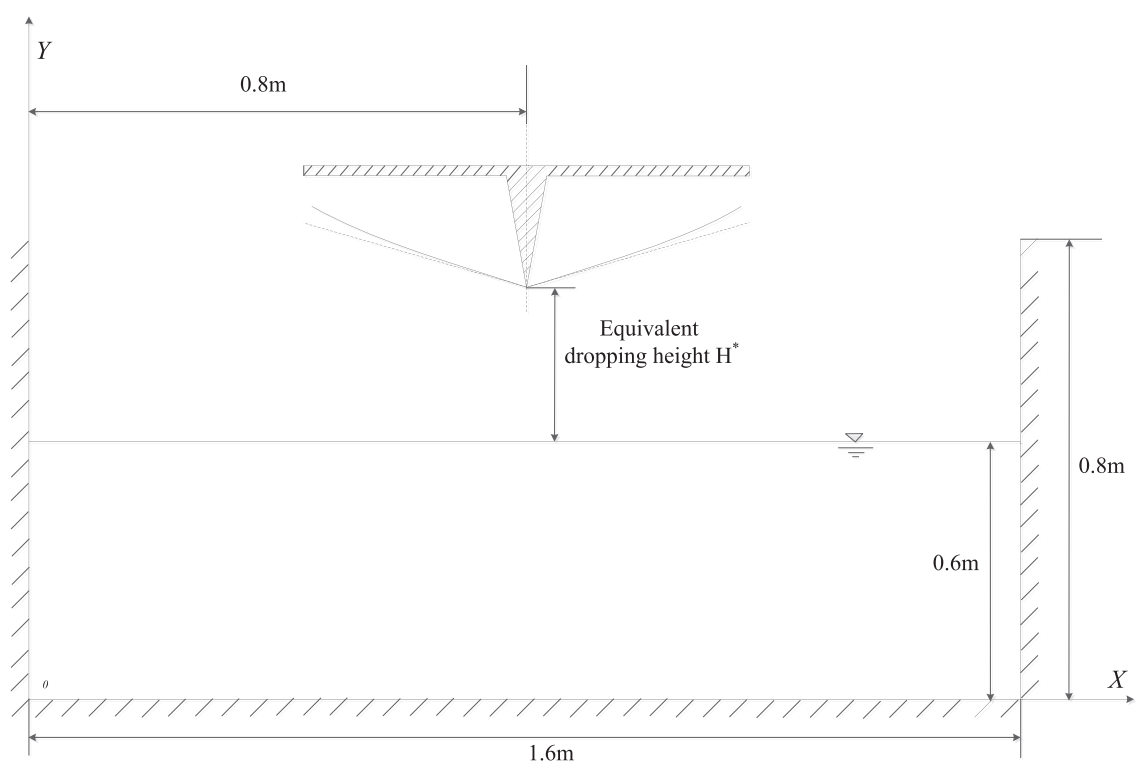

Fig. 5.5. Initial configuration of the wedge dropping problem.

Table 5.1

\begin{tabular}{llll}
\hline & Case 1 & Case 2 & Case 3 \\
\hline Material & E-Glass (woven)/epoxy & Aluminium \\
Young' modulus E $(\mathrm{GPa})$ & 30.3 & & 68 \\
Density $\rho\left(\mathrm{kg} / \mathrm{m}^{3}\right)$ & 2015 & 2700 \\
Mass of the rig $(\mathrm{kg} / \mathrm{m})$ & 22 & \\
Length of each bottom L $(\mathrm{m})$ & 0.3 & & \\
Thickness $(\mathrm{mm})$ & 2.2 & & 2 \\
Deadrise angle $\beta\left({ }^{\circ}\right)$ & 30 & 5.57 & 4 \\
Entry speed $(\mathrm{m} / \mathrm{s})$ & 4.29 & 1.5813 & 0.8155 \\
Equivalent Height $\mathrm{H}^{*}(\mathrm{~m})$ & 0.938 & & \\
\hline
\end{tabular}

that during the period after releasing from height $\mathrm{H}^{*}$ and before touching the free surface, the flexible bottom will also develop a small amount of deformation. Therefore, in order to make the simulation more consistent with the real experimental condition, the simulation is started from the releasing instant, as shown in Fig. 5.5.

In this study, three different flexible cases are simulated. The related parameters of the flexible wedge are listed in Table 5.1.

The first three modes are taken into account and the corresponding first three circular frequencies are: $\omega_{1}=96.2104, \omega_{2}=602.9434, \omega_{3}=1688.2579$.

The fluid field is discretised by particles with the initial space of $0.005 \mathrm{~m}$, which results in 38400 fluid particles (40122 particles in total). The time interval is determined by the CFL condition with a maximum limit of $0.0002 \mathrm{~s}$. With an average iteration number of 6.5 times for each FSI coupling time step, about 22 CPU hours' time are required for the simulation of 0.06 s physical duration using the same computer hardware, as described in Section 5.1 It is worth mentioning that the majority of the computational time is used for the fluid solver, i.e. MPS part. The time used for structure solver is neglectable considering the scale of the linear system is only $4 \times 4$. And only one time iteration is required for an order of $10^{-5}$ computational accuracy.

The accelerations of the flexible wedges that are calculated by the coupled Modified MPS and CRMS model are compared with the results from experiment (Panciroli, 2013), rigid-body simulation and Wagner's theory in Fig. 5.6, respectively. The rigid-body computation is conducted by the normal routine for 2D situation, as derived in Section 2.3.1.1. Since the motion in $X$ direction is also constrained due to the symmetry property as in flexible cases, only the equation corresponding to $Y$ part in Eq. (2.65) is required to be solved. 
(a)

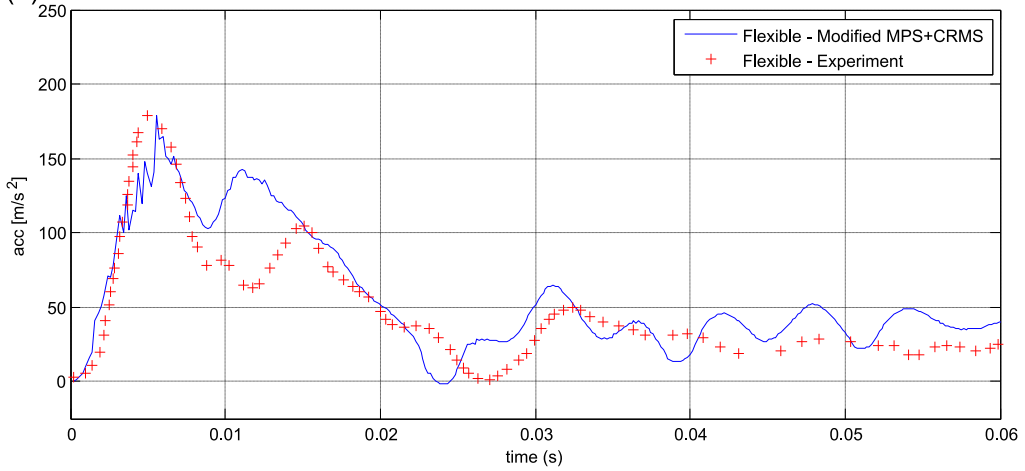

(b)

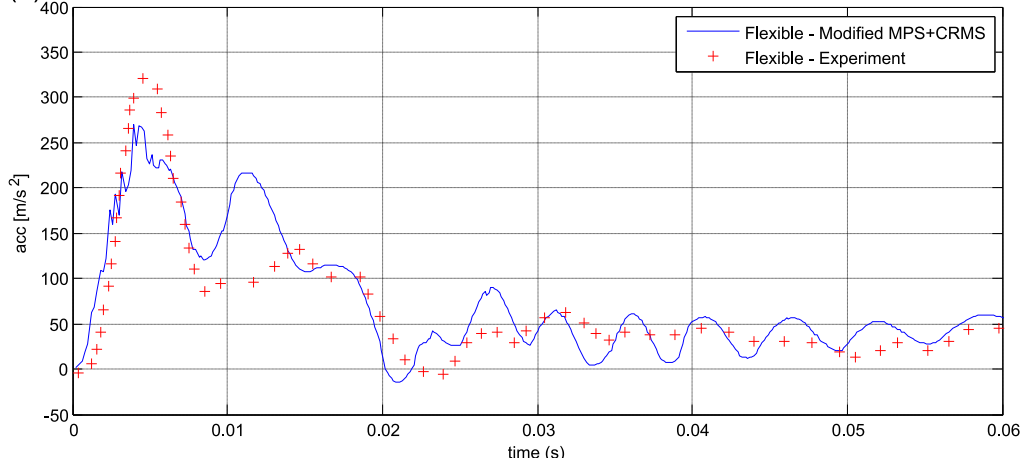

(c)

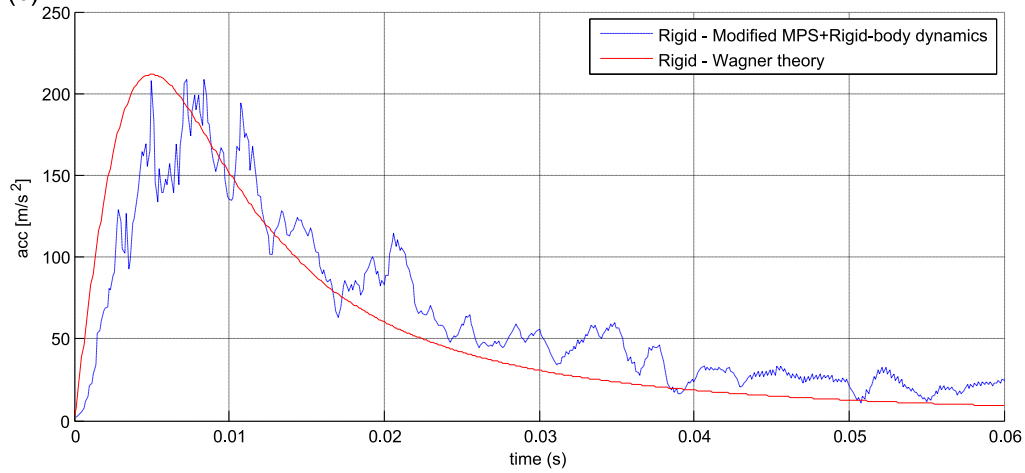

(d)

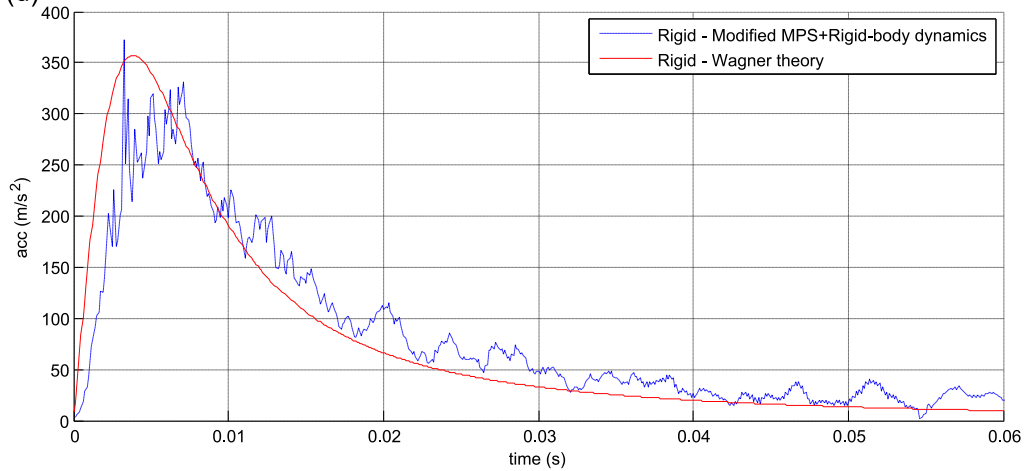

Fig. 5.6. The acceleration of the flexible/rigid wedge: (a) comparison for Case 1 between Modified MPS + CRMS and Experiment (Panciroli, 2013); (b) comparison for Case 2 between Modified MPS + CRMS and Experiment (Panciroli, 2013); (c) comparison for rigid wedge between Modified MPS + Rigidbody dynamics and Wagner's theory with the same entry speed as in Case $1(4.29 \mathrm{~m} / \mathrm{s}) ;(\mathrm{d})$ comparison for rigid wedge between Modified MPS +Rigid-body dynamics and Wagner's theory with the same entry speed as in Case $2(5.57 \mathrm{~m} / \mathrm{s})$. 

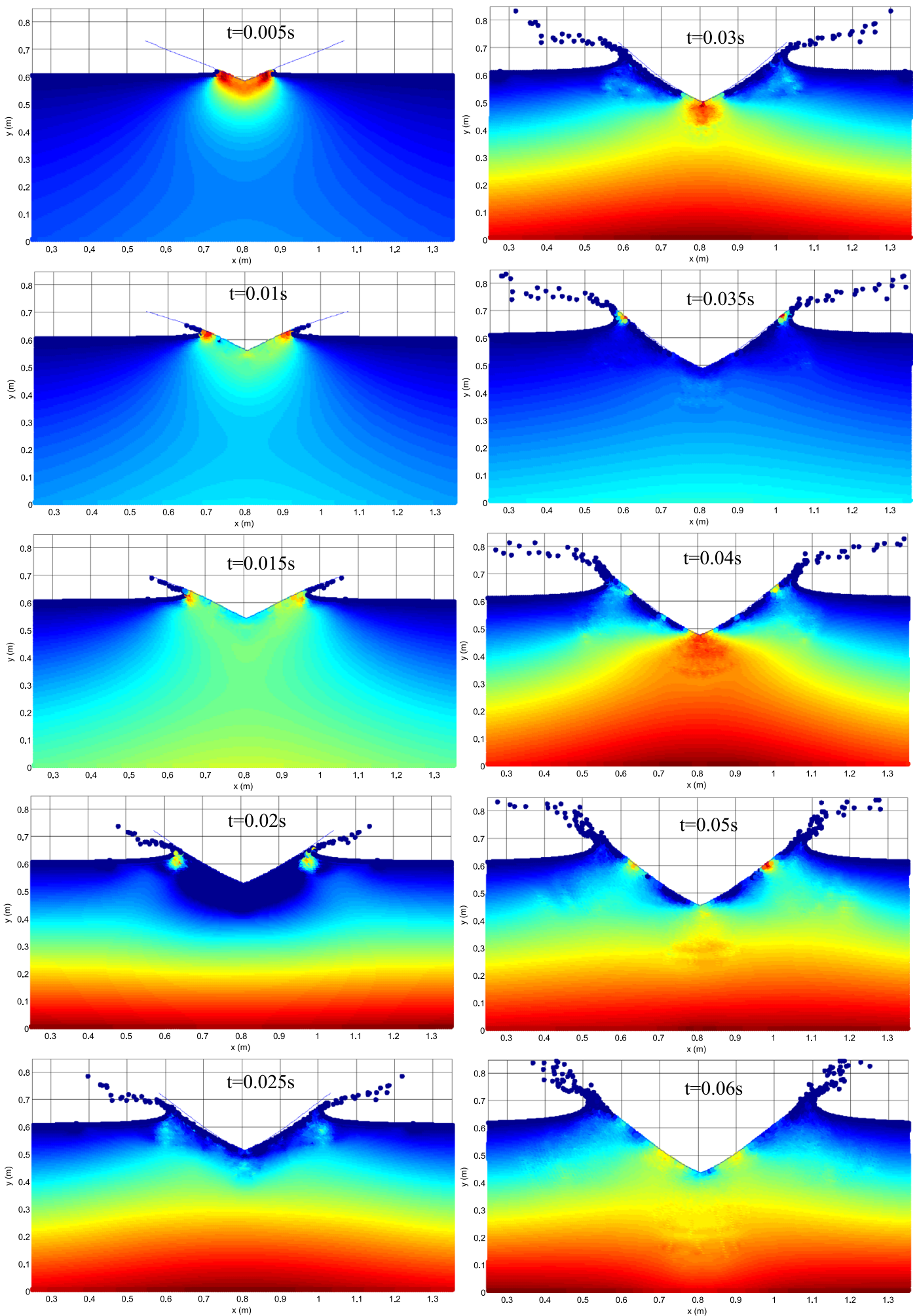

Fig. 5.7. The pressure contour at different time instants for Case 2 . 

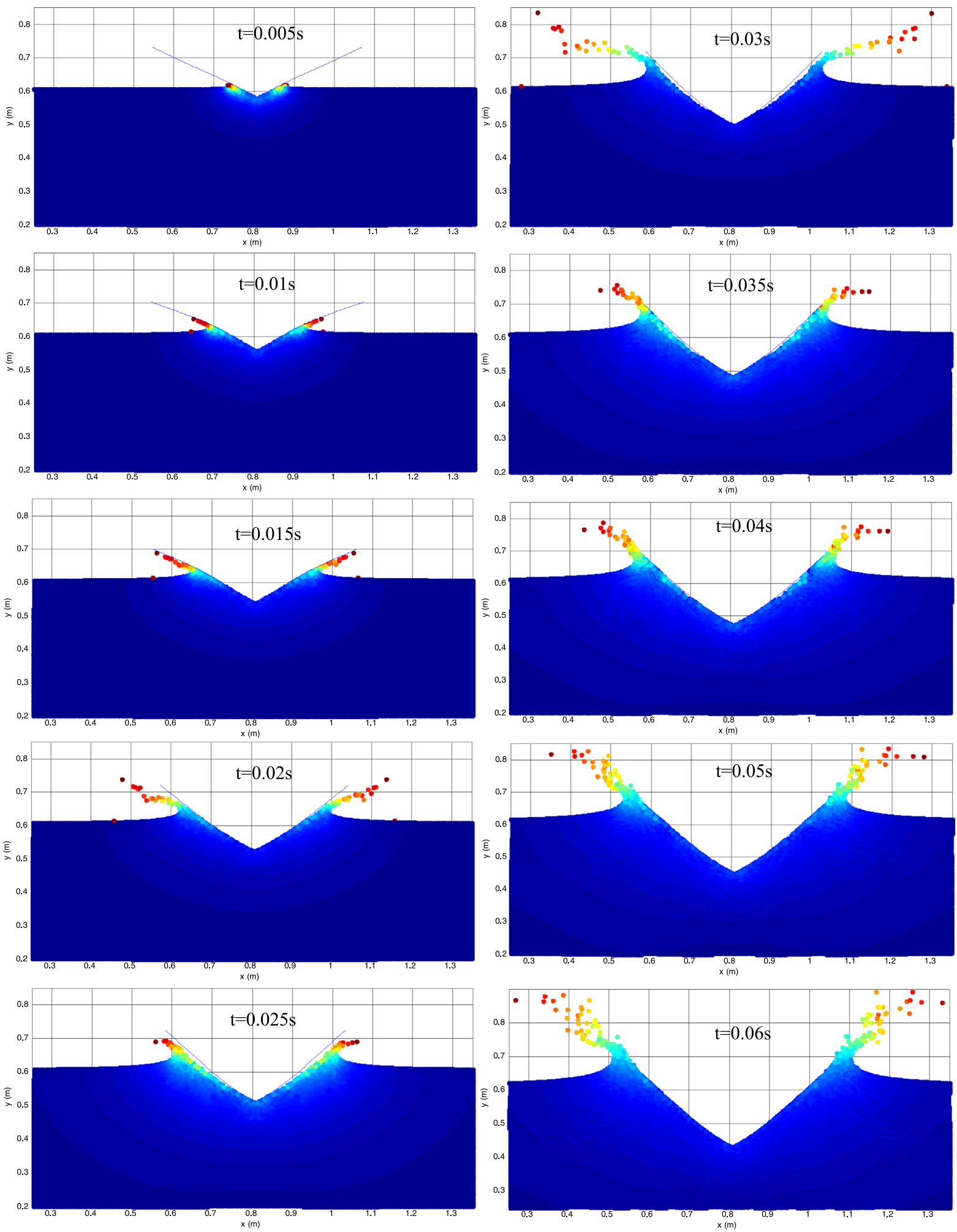

Fig. 5.8. The velocity contour at different time instants for Case 2 . 

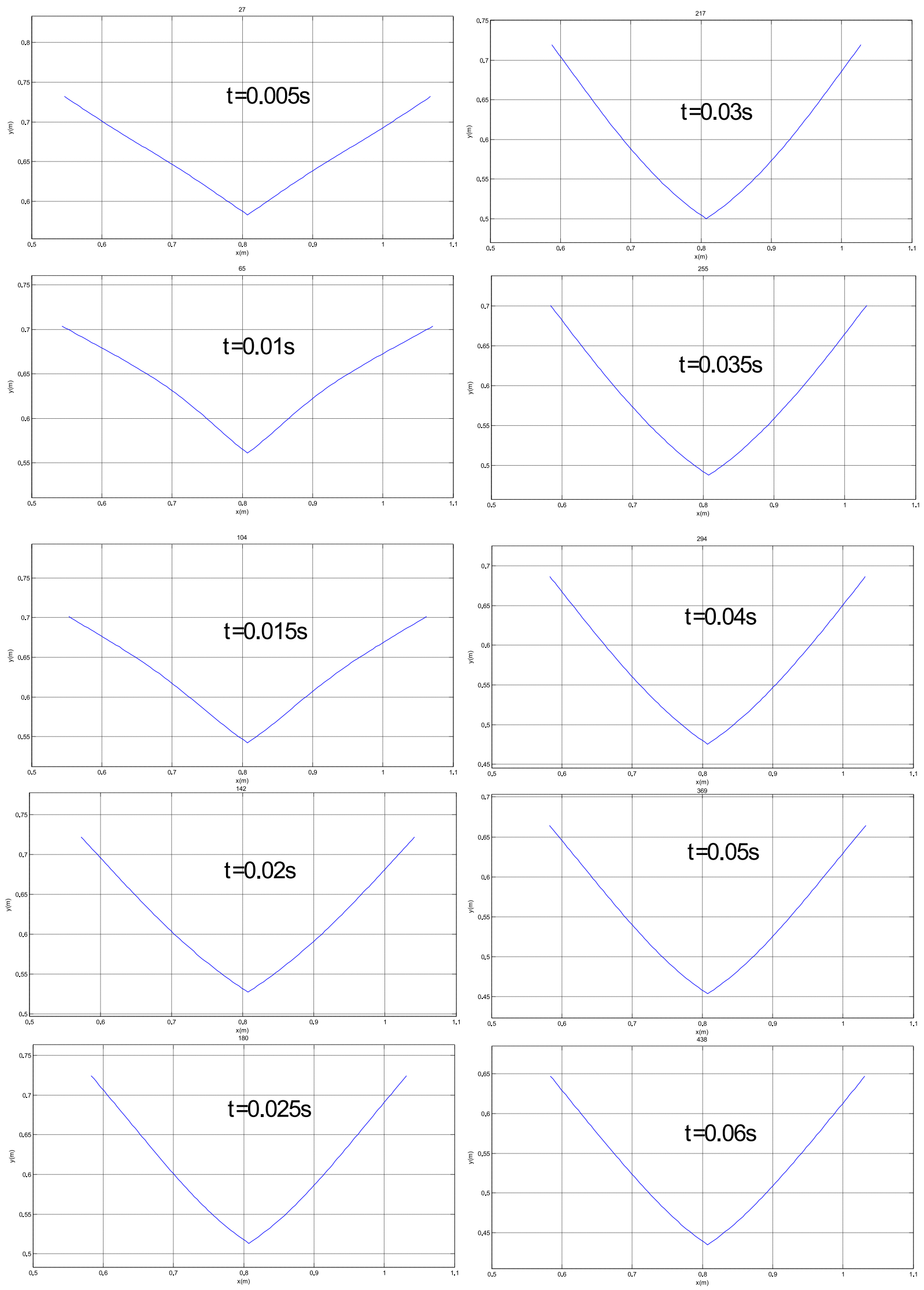

Fig. 5.9. Deformation of the flexible bottom at different time instants for Case 2. 
a

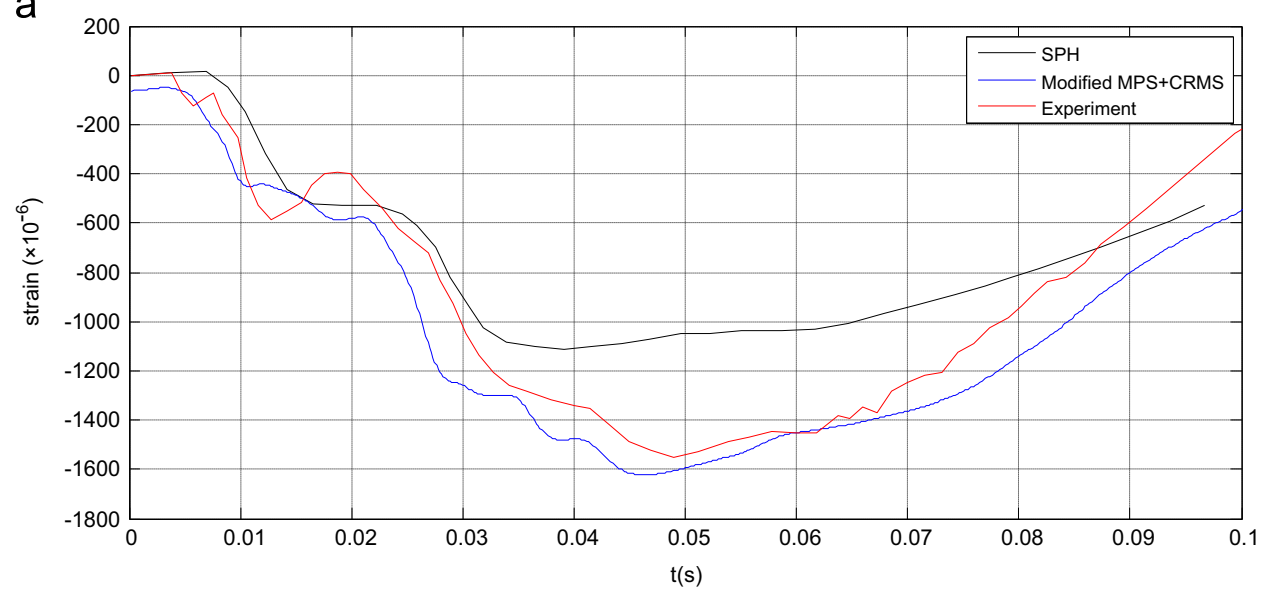

b

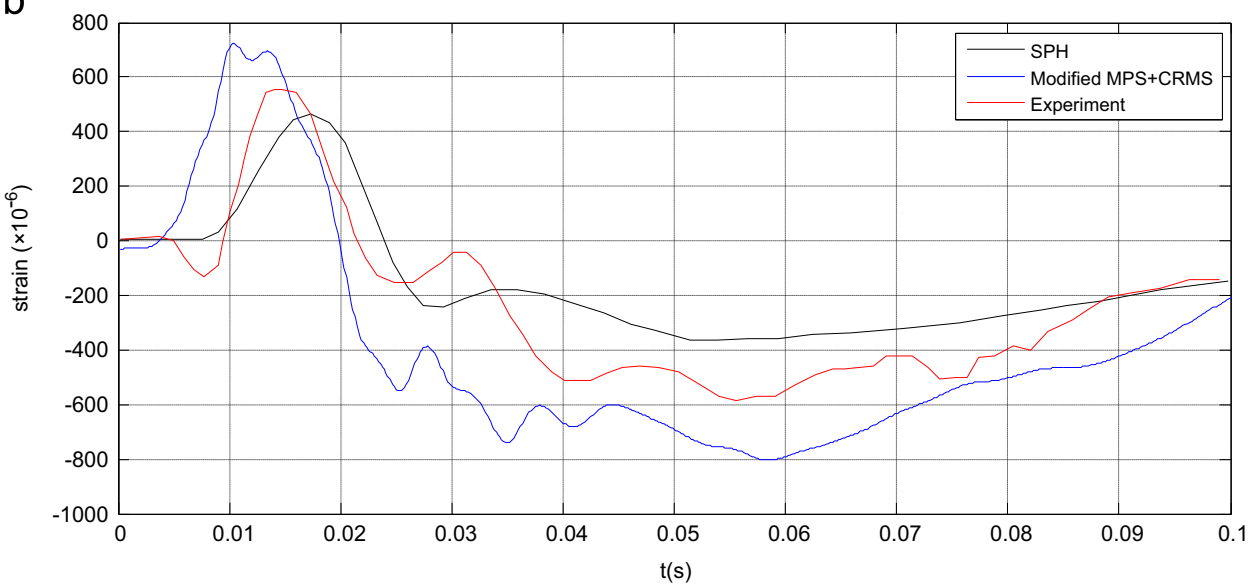

Fig. 5.10. The comparison of strain results for case 3 between experiment (Panciroli, 2013), Modified MPS + CRMS and SPH (Panciroli, 2013) at different positions ((a) and (b) corresponds to $30 \mathrm{~mm}$ and $120 \mathrm{~mm}$ from the wedge tip respectively).

As shown in Fig. 5.6(a) and (b), the numerical results coincide with the experimental data in terms of both the main trend and the first impact pressure peak time. In contrast to the rigid case in Fig. 5.6(c) and (d), there is a trough in both the experimental and numerical results for flexible cases in Fig. 5.6(a) and (b) (at about $0.025 \mathrm{~s}$ ). Another distinguished feature in flexible cases is that after the trough of the curve, the acceleration tends to oscillate around a constant value until the end of the simulation. The numerical model gives an overshot for the second peak acceleration value. And the peak time is also earlier than the experimental data. This is probably caused by the 2D limitation, since the water cannot be pushed aside along the tip direction like in real 3D environment and consequently the improper gathering water could generate a higher pressure. The difference of the dynamic characteristics between 2D beam assumption and the real 3D plate used in the experiment (e.g. the natural frequencies for the 3D plate is $\omega_{1}=123.72, \omega_{2}=775.35, \omega_{3}=217.11$ ) might be another reason of the deviation shown in Fig. 5.6(a) and (b).

The pressure and velocity contours are shown in Figs. 5.7 and 5.8, respectively. Due to the flexibility of the wedge bottom, the cavitation starts to develop from roughly $t=0.02 \mathrm{~s}$ and vanishes until about $t=0.04 \mathrm{~s}$. Because the current model only involves the water phase, the dynamics which is caused by the entrapped air between the wedge bottom and water could not be captured correctly. This is probably the reason that the numerical accelerations in Fig. 5.6(a) and (b) show a larger fluctuation during the cavitation period.

Fig. 5.9 shows the deformation of the flexible bottoms at some typical time instants. During the initial stage of the impact, the bottoms are bended by the coupling effect of the inertia and the concentrated impact force near the wedge tip. After about $t=0.02 \mathrm{~s}$, the deformation of the beam starts to bounce back towards the symmetry line of the wedge. This process is also reflected in the time history of strain that is monitored at two different locations on upper surface of each bottom, i.e. $30 \mathrm{~mm}$ and $120 \mathrm{~mm}$ away from the wedge tip respectively, as is shown in Fig. 5.10. The positive part of the strain in Fig. 5.10(b) (monitored at $120 \mathrm{~mm}$, which is about the middle of the bottom) represents the initial bending stage, as is 
shown in Fig. 5.9. After that, the strain remains negative because of the pressure of the surrounding water. The numerical strain record matches well with the experimental data.

\section{Conclusion}

The coupled MPS-modal superposition method proposed in this research has been successfully applied to simulate 2-D nonlinear water-structure interaction problems involving large rigid motions with small elastic structure deformation. For the structure model it is shown that it reduces to a small scale equations system (i.e., $6 \times 6$ or $4 \times 4$, as shown in the floating beam and flexible beam cases, respectively) regardless of the size of structure.

The numerical examples show that the developed numerical method is capable of providing stable and reasonable accurate results for simulating nonlinear FSI problems. For the violent FSI cases, the mutual effect between rigid-body motion and flexible deformation will affect the overall rigid-body motion pattern, e.g. the trajectory of the mass centre of the structure, to a non neglectable level. This feature suggests that the normal modal superposition approach, in which the flexible part is considered separately with rigid-body motion, is not enough for this kind of simulation. Moreover, if we take the efficiency of this model (compared with e.g. FE analysis) into account , the proposed method is quite suitable for the nonlinear FSI simulation of marine structures, which typically can be described as a large rigid attached with elastic beam-like, performing large overall rigid-body motion and small elastic deformation.

\section{Acknowledgements}

Authors acknowledge the Lloyd's Register Group, University of Southampton and China Scholarship Council to support this research. Also great thanks to Dr. R. Panciroli from University of Bologna, Italy, for his kind clarification of some experimental details; which are used for validation, in this paper.

\section{Appendix A}

For a variable $\zeta$ (i.e. each element in Eqs. (2.76)-(2.81)), its first and second order time derivatives $\dot{\zeta}_{n+1}, \ddot{\zeta}_{n+1}$ at next time stept $=t_{n+1}$, can be expressed using Newmark method as:

$$
\begin{aligned}
& \ddot{\zeta}_{n+1}=\frac{1}{\beta \Delta t^{2}} \zeta_{n+1}-\left[\frac{\zeta_{n}}{\beta \Delta t^{2}}+\frac{\dot{\zeta}_{n}}{\beta \Delta t}+\left(\frac{1}{2 \beta}-1\right) \ddot{\zeta}_{n}\right] \\
& \dot{\zeta}_{n+1}=\frac{\gamma}{\beta \Delta t} \zeta_{n+1}+\left(1-\frac{\gamma}{\beta}\right) \dot{\zeta}_{n}+\Delta t\left[(1-\gamma)-\gamma\left(\frac{1}{2 \beta}-1\right)\right] \ddot{\zeta}_{n}-\frac{\gamma}{\beta \Delta t} \zeta_{n}
\end{aligned}
$$

where $\gamma=\frac{1-2 \alpha}{2}, \beta=\frac{(1-\alpha)^{2}}{4}$, and $\alpha$ is chosen to be $\alpha=-0.05$ in this study.

To simplify the above equations, the following definitions are introduced:

$C_{1}=\frac{1}{\beta \Delta t^{2}}, C_{3}=\frac{\gamma}{\beta \Delta t}$

$C_{2 \zeta}=-\left[\frac{\zeta_{n}}{\beta \Delta t^{2}}+\frac{\dot{\zeta}_{n}}{\beta \Delta t}+\left(\frac{1}{2 \beta}-1\right) \ddot{\zeta}_{n}\right]$,

$C_{3 \zeta}=\left(1-\frac{\gamma}{\beta}\right) \dot{\zeta}_{n}+\Delta t\left[(1-\gamma)-\gamma\left(\frac{1}{2 \beta}-1\right)\right] \ddot{\zeta}_{n}-\frac{\gamma}{\beta \Delta t} \zeta_{n}$

in which $C_{2 \zeta}$ and $C_{3 \zeta}$ correspond to the particular variable $\zeta$

Using Newmark method to replace the velocity and acceleration terms with position values, the governing equations of floating beams (i.e. Eqs. (2.74)-(2.81)) then become

$$
\begin{aligned}
f_{1}= & M\left(C_{1} X_{c R}+C_{2 X_{c R}}\right)+\left(C_{3} \theta+C_{4 \theta}\right)^{2} \sin \theta\left(\psi_{01} q_{1}+\psi_{02} q_{2}+\psi_{03} q_{3}\right) \\
& -2\left(C_{3} \theta+C_{4 \theta}\right) \cos \theta\left[\psi_{01}\left(C_{3} q_{1}+C_{4 q_{1}}\right)+\psi_{02}\left(C_{3} q_{2}+C_{4 q_{2}}\right)+\psi_{03}\left(C_{3} q_{3}+C_{4 q_{3}}\right)\right] \\
& -\left(C_{1} \theta+C_{2 \theta}\right) \cos \theta\left(\psi_{01} q_{1}+\psi_{02} q_{2}+\psi_{03} q_{3}\right)-\sin \theta\left[\psi_{01}\left(C_{1} q_{1}+C_{2 q_{1}}\right)\right. \\
& \left.+\psi_{02}\left(C_{1} q_{2}+C_{2 q_{2}}\right)+\psi_{03}\left(C_{1} q_{3}+C_{2 q_{3}}\right)\right]-Q_{X_{c 1}}=0 \\
f_{2}= & M\left(C_{1} Y_{c R}+C_{2 Y_{c R}}\right)-\left(C_{3} \theta+C_{4 \theta}\right)^{2} \cos \theta\left(\psi_{01} q_{1}+\psi_{02} q_{2}+\psi_{03} q_{3}\right) \\
& -2\left(C_{3} \theta+C_{4 \theta}\right) \sin \theta\left[\psi_{01}\left(C_{3} q_{1}+C_{4 q_{1}}\right)+\psi_{02}\left(C_{3} q_{2}+C_{4 q_{2}}\right)+\psi_{03}\left(C_{3} q_{3}+C_{4 q_{3}}\right)\right] \\
& -\left(C_{1} \theta+C_{2 \theta}\right) \sin \theta\left(\psi_{01} q_{1}+\psi_{02} q_{2}+\psi_{03} q_{3}\right) \\
& +\cos \theta\left[\psi_{01}\left(C_{1} q_{1}+C_{2 q_{1}}\right)+\psi_{02}\left(C_{1} q_{2}+C_{2 q_{2}}\right)+\psi_{03}\left(C_{1} q_{3}+C_{2 q_{3}}\right)\right]+M g-Q_{X_{c 2}}=0, \\
f_{3}= & -\left[\left(C_{1} X_{c R}+C_{2 X_{c R}}\right) \cos \theta+\left(C_{1} Y_{c R}+C_{2 Y_{c R}}\right) \sin \theta\right]\left(\psi_{01} q_{1}+\psi_{02} q_{2}+\psi_{03} q_{3}\right) \\
& +\left(C_{1} \theta+C_{2 \theta}\right)\left(I_{f}+q_{1}{ }^{2}+q_{2}{ }^{2}+q_{3}{ }^{2}\right)+2\left(C_{3} \theta+C_{4 \theta}\right)\left[\left(C_{3} q_{1}+C_{4 q_{1}}\right) q_{1}+\left(C_{3} q_{2}+C_{4 q_{2}}\right) q_{2}+\left(C_{3} q_{3}+C_{4 q_{3}}\right) q_{3}\right]
\end{aligned}
$$


$+\left[\psi_{11}\left(C_{1} q_{1}+C_{2 q_{1}}\right)+\psi_{12}\left(C_{1} q_{2}+C_{2 q_{2}}\right)+\psi_{13}\left(C_{1} q_{3}+C_{2 q_{3}}\right)\right]-Q_{\theta}=0$,

$f_{4}=\left[-\left(C_{1} X_{c R}+C_{2 X_{c R}}\right) \sin \theta+\left(C_{1} Y_{c R}+C_{2 Y_{C R}}\right) \cos \theta\right] \psi_{01}+\left(C_{1} \theta+C_{2 \theta}\right) \psi_{11}-\left(C_{3} \theta+C_{4 \theta}\right)^{2} q_{1}+C_{1} q_{1}+C_{2 q_{1}}+\omega_{1}^{2} q_{1}-Q_{q_{1}}=0$, $f_{5}=\left[-\left(C_{1} X_{c R}+C_{2 X_{C R}}\right) \sin \theta+\left(C_{1} Y_{c R}+C_{2 Y_{C R}}\right) \cos \theta\right] \psi_{02}+\left(C_{1} \theta+C_{2 \theta}\right) \psi_{12}-\left(C_{3} \theta+C_{4 \theta}\right)^{2} q_{2}+C_{1} q_{2}+C_{2 q_{2}}+\omega_{2}^{2} q_{2}-Q_{q_{2}}=0$, $f_{6}=\left[-\left(C_{1} X_{c R}+C_{2 X_{c R}}\right) \sin \theta+\left(C_{1} Y_{c R}+C_{2 Y_{c R}}\right) \cos \theta\right] \psi_{03}+\left(C_{1} \theta+C_{2 \theta}\right) \psi_{13}-\left(C_{3} \theta+C_{4 \theta}\right)^{2} q_{3}+C_{1} q_{3}+C_{2 q_{3}}+\omega_{3}^{2} q_{3}-Q_{q_{3}}=0$,

These set of equations are solved by Newton-Raphson method. The elements of the Jacobi matrix are:

$\frac{\partial f_{1}}{\partial X_{c R}}=M C_{1}, \quad \frac{\partial f_{1}}{\partial Y_{c R}}=0$,

$\frac{\partial f_{1}}{\partial \theta}=\left(\psi_{01} q_{1}+\psi_{02} q_{2}+\psi_{03} q_{3}\right)\left[\left(C_{3} \theta+C_{4 \theta}\right)^{2} \cos \theta+2 C_{3}\left(C_{3} \theta+C_{4 \theta}\right) \sin \theta\right]$

$-2\left[\psi_{01}\left(C_{3} q_{1}+C_{4 q_{1}}\right)+\psi_{02}\left(C_{3} q_{2}+C_{4 q_{2}}\right)+\psi_{03}\left(C_{3} q_{3}+C_{4 q_{3}}\right)\right]\left[C_{3} \cos \theta-\left(C_{3} \theta+C_{4 \theta}\right) \sin \theta\right]$

$-\left(\psi_{01} q_{1}+\psi_{02} q_{2}+\psi_{03} q_{3}\right)\left[C_{1} \cos \theta-\left(C_{1} \theta+C_{2 \theta}\right) \sin \theta\right]$

$-\cos \theta\left[\psi_{01}\left(C_{1} q_{1}+C_{2 q_{1}}\right)+\psi_{02}\left(C_{1} q_{2}+C_{2 q_{2}}\right)+\psi_{03}\left(C_{1} q_{3}+C_{2 q_{3}}\right)\right]$,

$\frac{\partial f_{1}}{\partial q_{1}}=\psi_{01}\left(C_{3} \theta+C_{4 \theta}\right)^{2} \sin \theta-2 \psi_{01} C_{3}\left(C_{3} \theta+C_{4 \theta}\right) \cos \theta-\psi_{01}\left(C_{1} \theta+C_{2 \theta}\right) \cos \theta-C_{1} \psi_{01} \sin \theta$,

$\frac{\partial f_{1}}{\partial q_{2}}=\psi_{02}\left(C_{3} \theta+C_{4 \theta}\right)^{2} \sin \theta-2 \psi_{02} C_{3}\left(C_{3} \theta+C_{4 \theta}\right) \cos \theta-\psi_{02}\left(C_{1} \theta+C_{2 \theta}\right) \cos \theta-C_{1} \psi_{02} \sin \theta$,

$\frac{\partial f_{1}}{\partial q_{3}}=\psi_{03}\left(C_{3} \theta+C_{4 \theta}\right)^{2} \sin \theta-2 \psi_{03} C_{3}\left(C_{3} \theta+C_{4 \theta}\right) \cos \theta-\psi_{03}\left(C_{1} \theta+C_{2 \theta}\right) \cos \theta-C_{1} \psi_{03} \sin \theta$,

$\frac{\partial f_{2}}{\partial X_{c R}}=0, \quad \frac{\partial f_{2}}{\partial Y_{c R}}=M C_{1}$,

$\frac{\partial f_{2}}{\partial \theta}=\left(\psi_{01} q_{1}+\psi_{02} q_{2}+\psi_{03} q_{3}\right)\left[\left(C_{3} \theta+C_{4 \theta}\right)^{2} \sin \theta-2 C_{3}\left(C_{3} \theta+C_{4 \theta}\right) \cos \theta\right]$

$-2\left[\psi_{01}\left(C_{3} q_{1}+C_{4 q_{1}}\right)+\psi_{02}\left(C_{3} q_{2}+C_{4 q_{2}}\right)+\psi_{03}\left(C_{3} q_{3}+C_{4 q_{3}}\right)\right]\left[C_{3} \sin \theta+\left(C_{3} \theta+C_{4 \theta}\right) \cos \theta\right]$

$-\left(\psi_{01} q_{1}+\psi_{02} q_{2}+\psi_{03} q_{3}\right)\left[C_{1} \sin \theta+\left(C_{1} \theta+C_{2 \theta}\right) \cos \theta\right]$

$-\sin \theta\left[\psi_{01}\left(C_{1} q_{1}+C_{2 q_{1}}\right)+\psi_{02}\left(C_{1} q_{2}+C_{2 q_{2}}\right)+\psi_{03}\left(C_{1} q_{3}+C_{2 q_{3}}\right)\right]$,

$\frac{\partial f_{2}}{\partial q_{1}}=-\psi_{01}\left(C_{3} \theta+C_{4 \theta}\right)^{2} \cos \theta-2 \psi_{01} C_{3}\left(C_{3} \theta+C_{4 \theta}\right) \sin \theta-\psi_{01}\left(C_{1} \theta+C_{2 \theta}\right) \sin \theta+C_{1} \psi_{01} \cos \theta$,

$\frac{\partial f_{2}}{\partial q_{2}}=-\psi_{02}\left(C_{3} \theta+C_{4 \theta}\right)^{2} \cos \theta-2 \psi_{02} C_{3}\left(C_{3} \theta+C_{4 \theta}\right) \sin \theta-\psi_{02}\left(C_{1} \theta+C_{2 \theta}\right) \sin \theta+C_{1} \psi_{02} \cos \theta$,

$\frac{\partial f_{2}}{\partial q_{3}}=-\psi_{03}\left(C_{3} \theta+C_{4 \theta}\right)^{2} \cos \theta-2 \psi_{03} C_{3}\left(C_{3} \theta+C_{4 \theta}\right) \sin \theta-\psi_{03}\left(C_{1} \theta+C_{2 \theta}\right) \sin \theta+C_{1} \psi_{03} \cos \theta$,

$\frac{\partial f_{3}}{\partial X_{c R}}=-C_{1} \cos \theta\left(\psi_{01} q_{1}+\psi_{02} q_{2}+\psi_{03} q_{3}\right), \frac{\partial f_{3}}{\partial Y_{c R}}=-C_{1} \sin \theta\left(\psi_{01} q_{1}+\psi_{02} q_{2}+\psi_{03} q_{3}\right)$,

$\frac{\partial f_{3}}{\partial \theta}=-\left[-\left(C_{1} X_{c R}+C_{2 X_{c R}}\right) \sin \theta+\left(C_{1} Y_{c R}+C_{2 Y_{C R}}\right) \cos \theta\right]\left(\psi_{01} q_{1}+\psi_{02} q_{2}+\psi_{03} q_{3}\right)$

$+C_{1}\left(I_{f}+q_{1}{ }^{2}+q_{2}{ }^{2}+q_{3}{ }^{2}\right)+2 C_{3}\left[\left(C_{3} q_{1}+C_{4 q_{1}}\right) q_{1}+\left(C_{3} q_{2}+C_{4 q_{2}}\right) q_{2}+\left(C_{3} q_{3}+C_{4 q_{3}}\right) q_{3}\right]$,

$\frac{\partial f_{3}}{\partial q_{1}}=-\psi_{01}\left[\left(C_{1} X_{c R}+C_{2 X_{c R}}\right) \cos \theta+\left(C_{1} Y_{c R}+C_{2 Y_{c R}}\right) \sin \theta\right]+2 q_{1}\left(C_{1} \theta+C_{2 \theta}\right)+2\left(C_{3} \theta+C_{4 \theta}\right)\left(2 C_{3} q_{1}+C_{4 q_{1}}\right)+\psi_{11} C_{1}$,

$\frac{\partial f_{3}}{\partial q_{2}}=-\psi_{02}\left[\left(C_{1} X_{c R}+C_{2 X_{c R}}\right) \cos \theta+\left(C_{1} Y_{c R}+C_{2 Y_{c R}}\right) \sin \theta\right]+2 q_{2}\left(C_{1} \theta+C_{2 \theta}\right)+2\left(C_{3} \theta+C_{4 \theta}\right)\left(2 C_{3} q_{2}+C_{4 q_{2}}\right)+\psi_{12} C_{1}$,

$\frac{\partial f_{3}}{\partial q_{3}}=-\psi_{03}\left[\left(C_{1} X_{c R}+C_{2 X_{c R}}\right) \cos \theta+\left(C_{1} Y_{c R}+C_{2 Y_{c R}}\right) \sin \theta\right]+2 q_{3}\left(C_{1} \theta+C_{2 \theta}\right)+2\left(C_{3} \theta+C_{4 \theta}\right)\left(2 C_{3} q_{3}+C_{4 q_{3}}\right)+\psi_{13} C_{1}$,

$\frac{\partial f_{4}}{\partial X_{c R}}=-C_{1} \psi_{01} \sin \theta, \frac{\partial f_{4}}{\partial Y_{c R}}=C_{1} \psi_{01} \cos \theta$,

$\frac{\partial f_{4}}{\partial \theta}=-\left[\left(C_{1} X_{c R}+C_{2 X_{c R}}\right) \cos \theta+\left(C_{1} Y_{c R}+C_{2 Y_{c R}}\right) \sin \theta\right] \psi_{01}+C_{1} \psi_{11}-2 C_{3} q_{1}\left(C_{3} \theta+C_{4 \theta}\right)$

$\frac{\partial f_{4}}{\partial q_{1}}=C_{1}+\omega_{1}^{2}-\left(C_{3} \theta+C_{4 \theta}\right)^{2}, \frac{\partial f_{4}}{\partial q_{2}}=\frac{\partial f_{4}}{\partial q_{3}}=0$,

$\frac{\partial f_{5}}{\partial X_{c R}}=-C_{1} \psi_{02} \sin \theta, \frac{\partial f_{5}}{\partial Y_{c R}}=C_{1} \psi_{02} \cos \theta$,

$\frac{\partial f_{5}}{\partial \theta}=-\left[\left(C_{1} X_{c R}+C_{2 X_{c R}}\right) \cos \theta+\left(C_{1} Y_{c R}+C_{2 Y_{c R}}\right) \sin \theta\right] \psi_{02}+C_{1} \psi_{12}-2 C_{3} q_{2}\left(C_{3} \theta+C_{4 \theta}\right)$

$\frac{\partial f_{5}}{\partial q_{2}}=C_{1}+\omega_{2}^{2}-\left(C_{3} \theta+C_{4 \theta}\right)^{2}, \frac{\partial f_{5}}{\partial q_{1}}=\frac{\partial f_{5}}{\partial q_{3}}=0$,

$\frac{\partial f_{6}}{\partial X_{c R}}=-C_{1} \psi_{03} \sin \theta, \frac{\partial f_{6}}{\partial Y_{c R}}=C_{1} \psi_{03} \cos \theta$, 


$$
\begin{aligned}
& \frac{\partial f_{6}}{\partial \theta}=-\left[\left(C_{1} X_{c R}+C_{2 X_{C R}}\right) \cos \theta+\left(C_{1} Y_{c R}+C_{2 Y_{C R}}\right) \sin \theta\right] \psi_{03}+C_{1} \psi_{13}-2 C_{3} q_{3}\left(C_{3} \theta+C_{4 \theta}\right) \\
& \frac{\partial f_{6}}{\partial q_{3}}=C_{1}+\omega_{3}^{2}-\left(C_{3} \theta+C_{4 \theta}\right)^{2}, \frac{\partial f_{6}}{\partial q_{1}}=\frac{\partial f_{6}}{\partial q_{2}}=0
\end{aligned}
$$

Similarly, the governing equations for the wedge with flexible bottom (Eqs. (2.92)-(2.95)) and its Jacobi matrix are:

$$
\begin{aligned}
& f_{1}=\left(2 M_{f}+M_{R}\right)\left(C_{1} Y_{c R}+C_{2 Y_{c R}}\right)+2 \cos \theta\left(\psi_{01}\left(C_{1} q_{1}+C_{2 q_{1}}\right)+\psi_{02}\left(C_{1} q_{2}+C_{2 q_{2}}\right)+\psi_{03}\left(C_{1} q_{3}+C_{2 q_{3}}\right)\right)+\left(2 M_{f}+M_{R}\right) g-Q_{X_{c 2}}=0 \\
& f_{2}=2\left(C_{1} Y_{c R}+C_{2 Y_{c R}}\right) \cos \theta \psi_{01}+2\left(C_{1} q_{1}+C_{2 q_{1}}\right)+2 \omega_{1}^{2} q_{1}-Q_{q_{1}}=0 \\
& f_{3}=2\left(C_{1} Y_{c R}+C_{2 Y_{c R}}\right) \cos \theta \psi_{02}+2\left(C_{1} q_{2}+C_{2 q_{2}}\right)+2 \omega_{2}^{2} q_{2}-Q_{q_{2}}=0 \\
& f_{4}=2\left(C_{1} Y_{c R}+C_{2 Y_{c R}}\right) \cos \theta \psi_{03}+2\left(C_{1} q_{3}+C_{2 q_{3}}\right)+2 \omega_{3}^{2} q_{3}-Q_{q_{3}}=0 \\
& \frac{\partial f_{1}}{\partial Y_{c R}}=\left(2 M_{f}+M_{R}\right) C_{1} \\
& \frac{\partial f_{1}}{\partial q_{1}}=2 C_{1} \psi_{01} \cos \theta \\
& \frac{\partial f_{1}}{\partial q_{2}}=2 C_{1} \psi_{02} \cos \theta \\
& \frac{\partial f_{1}}{\partial q_{3}}=2 C_{1} \psi_{03} \cos \theta \\
& \frac{\partial f_{2}}{\partial Y_{c R}}=2 C_{1} \psi_{01} \cos \theta \\
& \frac{\partial f_{2}}{\partial q_{1}}=2 C_{1}+2 \omega_{1}^{2}, \frac{\partial f_{2}}{\partial q_{2}}=\frac{\partial f_{2}}{\partial q_{3}}=0 \\
& \frac{\partial f_{3}}{\partial Y_{c R}}=2 C_{1} \psi_{02} \cos \theta, \\
& \frac{\partial f_{3}}{\partial q_{2}}=2 C_{1}+2 \omega_{2}^{2}, \frac{\partial f_{3}}{\partial q_{1}}=\frac{\partial f_{3}}{\partial q_{3}}=0 \\
& \frac{\partial f_{4}}{\partial Y_{c R}}=2 C_{1} \psi_{03} \cos \theta \\
& \frac{\partial f_{4}}{\partial q_{3}}=2 C_{1}+2 \omega_{3}^{2}, \frac{\partial f_{4}}{\partial q_{1}}=\frac{\partial f_{4}}{\partial q_{2}}=0
\end{aligned}
$$

\section{References}

Akimoto, H., 2013. Numerical simulation of the flow around a planing body by MPS method. Ocean Engineering 64, 72-79.

Bishop, R.E.D., Price, W.G., Wu, Y., 1986. A General Linear Hydroelasticity Theory of Floating Structures Moving in a Seaway. Philosophical Transactions of the Royal Society A: Mathematical, Physical and Engineering Sciences 316, 375-426.

Brown, D.L., Cortez, R., Minion, M.L., 2001. Accurate Projection Methods for the Incompressible Navier-Stokes Equations. Journal of Computational Physics $168,464-499$.

Chen, X.-j, Wu, Y.-s, Cui, W.-c, Jensen, J.J., 2006. Review of hydroelasticity theories for global response of marine structures. Ocean Engineering 33, $439-457$. Chorin, A.J., 1967. A numerical method for solving incompressible viscous flow problems. Journal of Computational Physics 2, $12-26$.

Crespo, A.J.C., 2008. Application of the Smoothed Particle Hydrodynamics model SPHysics to free-surface hydrodynamics PhD thesis. Universidade De Vigo.

Jin, J.Z., Xing, J.T., 2007. Transient dynamic analysis of a floating beam-water interaction system excited by the impact of a landing beam. Journal of Sound and Vibration 303, 371-390.

Koshizuka, S., Oka, Y., 1996. Moving-Particle Semi-Implicit Method for Fragmentation of incompressible Fluid. Nuclear Science and Engineering 123, $421-434$.

Khayyer, A., Gotoh, H., 2009. Modified Moving Particle Semi-implicit methods for the prediction of 2D wave impact pressure. Coastal Engineering 56, 419-440.

Khayyer, A., Gotoh, H., 2013. Enhancement of performance and stability of MPS mesh-free particle method for multiphase flows characterized by high density ratios. Journal of Computational Physics 242, 211-233.

Khayyer, A., Gotoh, H., 2011. Enhancement of stability and accuracy of the moving particle semi-implicit method. Journal of Computational Physics 230, 3093-3118.

Kashiwagi, M., 2000. A time-domain mode-expansion method for calculating transient elastic responses of a pontoon-type VLFS. Journal of Marine Science and Technology 5, 89-100.

Koh, C.G., Gao, M., Luo, C., 2012. A new particle method for simulation of incompressible free surface flow problems. International Journal for Numerical Methods in Engineering 89, 1582-1604.

Lee, B.H., Park, J.C., Kim, M.H., Hwang, S.C., 2011. Step-by-step improvement of MPS method in simulating violent free-surface motions and impact-loads. Computer Methods in Applied Mechanics and Engineering 200, 1113-1125.

Lee, C.J.K., Noguchi, H., Koshizuka, S., 2007. Fluid-shell structure interaction analysis by coupled particle and finite element method. Computers Structures $85,688-697$.

Monaghan, 1994. Simulating Free Surface Flows with SPH. Journal of Computational Physics 110, $399-406$.

Newman, J.N., 1994. Wave effect on deformable bodies. Applied Ocean Research 16, 47-59.

Newmark, N.M., 1959. A Method of Computation for Structural Dynamics. Journal of the Engineering Mechanics Division 85, 67-94.

Panciroli, R., 2013. Hydroelastic Impacts of Deformable Wedges. Solid Mechanics and Its Applications 192, 1-45.

Z. Sun, K. Djidjeli, J. T. Xing, F. Cheng, and A. Javed, "Some modifications of MPS method for incompressible free surface flow " presented at the 11th World Congress on Computational Mechanics (WCCM XI), Barcelona, Spain, 2014.

Sun, Z., Djidjeli, K., Xing, J.T., Cheng, F., 2015. Modified MPS method for the 2D Fluid Structure Interaction problems with free surface. Computers and Fluids $122,47-65$. 
Shibata, K., Koshizuka, S., Tanizawa, K., 2009. Three-dimensional numerical analysis of shipping water onto a moving ship using a particle method. Journal of Marine Science and Technology 14, 214-227.

Shibata, K., Koshizuka, S., Sakai, M., Tanizawa, K., 2012. Lagrangian simulations of ship-wave interactions in rough seas. Ocean Engineering $42,13-25$.

Sun, H., 2007. A Boundary Element Method Applied to Strongly Nonlinear Wave-Body Interaction Problems PhD thesis. Norwegian University of Science and Technology.

Tsuruta, N., Khayyer, A., Gotoh, H., 2013. A Short Note on Dynamic Stabilization of Moving Particle Semi-implicit Method. Computers Fluids 82, 158-164.

Temarel, P., Hirdaris, S.E., 2009. Hydroelasticity of ships: recent advances and future trends. Proceedings of the Institution of Mechanical Engineers, Part Megye: Journal of Engineering for the Maritime Environment 223, 305-330. 